\title{
Reliability analysis of a complex system with hybrid structures and multi-level
}

\section{dependent life metrics}

\author{
Lechang Yanga ${ }^{\mathrm{a}, \mathrm{b}, 1}$, Pidong Wang ${ }^{\mathrm{a}}$, Qiang Wang ${ }^{\mathrm{a}}$, Sifeng $\mathrm{Bi}^{\mathrm{c}}$, Rui Peng ${ }^{\mathrm{d}}$, Jasper Behrensdorf ${ }^{\mathrm{b}}$ and \\ Michael Beer ${ }^{b, e, f}$ \\ ${ }^{a}$ School of Mechanical Engineering, University of Science and Technology Beijing, Beijing 100083, China \\ ${ }^{b}$ Institute for Risk and Reliability, Leibniz University Hannover, Hannover 30167, Germany \\ c School of Aerospace Engineering, Beijing Institute of Technology, Beijing 100081, China \\ ${ }^{d}$ School of Economics \& Management, Beijing University of Technology, Beijing 100124, China \\ ' Institute for Risk and Uncertainty, University of Liverpool, Liverpool L69 7ZF, United Kingdom \\ ${ }^{f}$ International Joint Research Center for Engineering Reliability and Stochastic Mechanics, Tongji University, Shanghai
} 200092, China

\begin{abstract}
In practical engineering, the presence of dependent evidence is not rare due to various imperfections. Misuse of such information in reliability analysis will lead to conflicting or even erroneous results. In this paper, we propose a Bayesian reliability approach for complex systems with dependent life metrics. Notions such as explicit evidence and implicit evidence are established based on an identification of different roles of multiple dependent evidence in the likelihood construction. A likelihood decomposition method is developed to convert the overall likelihood into a product of Explicit Evidence-based Likelihood (EEL) function and Implicit Evidence-based Likelihood (IEL) function. An inferential diagram is developed to intuitively generate the required implicit evidence taking both outer-source information and the system configuration into consideration. An algorithm is then presented for implementation. The contribution of our work is a systematic investigation of the role of dependent evidence in system reliability evaluation and a full Bayesian approach that is applied to various system reliability models.
\end{abstract} Extensive numerical cases and a practical engineering case are demonstrated for validation and to illustrate the benefits of our approach.

\footnotetext{
1 Corresponding author.

E-mail: yanglechang@ustb.edu.cn
} 


\section{Key words}

Reliability analysis; Bayesian network; Dependent life metrics; Hybrid structure; Multi-source conflicting information; Likelihood function

Acronyms and Notations

\begin{tabular}{|l|l|l|l|}
\hline RBD & Reliability Block Diagram & $\mathbf{A}_{j}$ & Predecessor nodes set of $X_{j}$ \\
\hline FT & Fault Tree & $\mathbf{Q}_{j}$ & Direct predecessor nodes set of $X_{j}$ \\
\hline PDF & Probability density Function & $\mathbf{C}$ & Component nodes set \\
\hline CDF & Cumulative Density Function & $\mathbf{S}$ & Subsystem/system nodes set \\
\hline BN & Bayesian Network & $\mathbf{O}$ & Observed nodes set \\
\hline DAG & Directed Acyclic Graph & $\mathbf{I}$ & Inferential nodes set \\
\hline CPT & Conditional Probability Table & $\mathbf{U}$ & Set of nodes that the likelihood of which is EEL \\
\hline EEL & Explicit Evidence-based Likelihood & $\mathbf{V}$ & Set of nodes that the likelihood of which is IEL \\
\hline IEL & Implicit Evidence-based Likelihood & $\boldsymbol{\theta}$ & Life time distribution parameter vector \\
\hline & & $\mathbf{p}$ & Conditional probability table parameters vector \\
\hline
\end{tabular}

\section{Introduction}

Reliability assessment of a system prototype at its early stage is of great importance since any later modification to the system design is costly, time-consuming and even impossible [1]. In reliability engineering practice, analyzing such a system is challenging due to various imperfections, e.g. undiscovered failure mechanisms, biased prior knowledge, undetermined system structure and limited test data [2]-[4]. In this scenario, it is desired to aggregate all available information to produce a more reliable result [5].

In recent years, the Bayesian-based approach has gained great attention from the reliability community as it provides a rigorous statistical model to integrate both expert judgments and experimental data [6]-[9]. In particular, Bayesian Networks (BN) have been increasingly employed in system reliability modeling and analysis [10]. Compared with traditional Reliability Block Diagram (RBD) or Fault Tree (FT) models, BNs are capable of incorporating both model uncertainty and parameter uncertainty in a coherent framework [11]. This capability is of great use in reliability modeling for prototypical systems because the interactive relationships between components 
have not been fully discovered yet. Model inherent uncertainty makes representing the system structure of this type of reliability model by "AND", "OR" gates unrealistic. Instead, by representing the reliability structure in conditional probabilities and assigning appropriate prior distributions, the BN parameters (both model parameters and distribution parameters) are progressively learned via Bayesian updating based on the accumulated evidence.

Besides the model uncertainty, another barrier in reliability analysis and assessment is insufficient test data. In practical engineering, repeatedly conducting independent reliability tests of the whole system is prohibitively expensive or not permissible. In order to increase the effectiveness of tests, it is not uncommon to install multiple sensors to monitor the health state of both subsystems and components and to collect data within one reliability experiment. Admitting the fact that component-level data is a valuable complement to the scarce system-level data, its misuse will, however, lead to conflicting or even erroneous results as they are essentially dependent. In this situation, a system reliability approach which is capable of correctly and efficiently dealing with dependent data has profound meanings to reliability practitioners.

The primary goal of this paper is to develop a Bayesian-based approach for system reliability analysis, where the model uncertainty and data dependence are particularly addressed. The dependent data discussed in this paper is based on continuous life metrics. We use the term "life metric" because two types of evidences are considered: (1) failure time data, which corresponds to a witness of failure occurring at an explicit time-point; (2) censored data, which suggests the exact failure time is not detected and we only know the failure time falls within an interval. The dependence discussed in this paper relies on the following two criteria:

(i) Coherence: the data set is drawn from the same coherent system, i.e. reliability block, fault tree, Bayesian network, etc.; and

(ii) Simultaneity: the data set is collected within the same period time.

The remainder of this paper is organized as follows. In Section 2, we demonstrate the problem at stake and 
review the relevant state-of-the-art methodologies. Sections 3 gives some preliminary knowledge. In section 4, the main body of our methodology is elaborated, followed by the developed algorithm for implementation. In section 5 , we validate the proposed method through three comparisons to well-established approaches. In section 6 , we demonstrate the benefits of our approach via an application to a case that existing methods have difficulties to handle. Finally, some concluding remarks are drawn in section 7.

\section{Problem description and state-of-the-art methodologies}

2.1 Dependent data in system reliability analysis

We demonstrate the significant importance of distinguishing dependent data and independent data in system reliability analysis through a simple motivating example.

Example 1 Consider the parallel system shown in Fig. 1, where two sensors are installed to record the failure time of the system or component. It is assumed that the time-to-failure of two components follows exponential distributions that are conditioned on the parameter $\lambda_{1}$ and $\lambda_{2}$ respectively. If we observed that component 1 is failed at $t_{1}$ first and then the system is failed at $t_{s}$. The task is to estimate the unknown parameters $\lambda_{1}, \lambda_{2}$ and system reliability using collected test data.

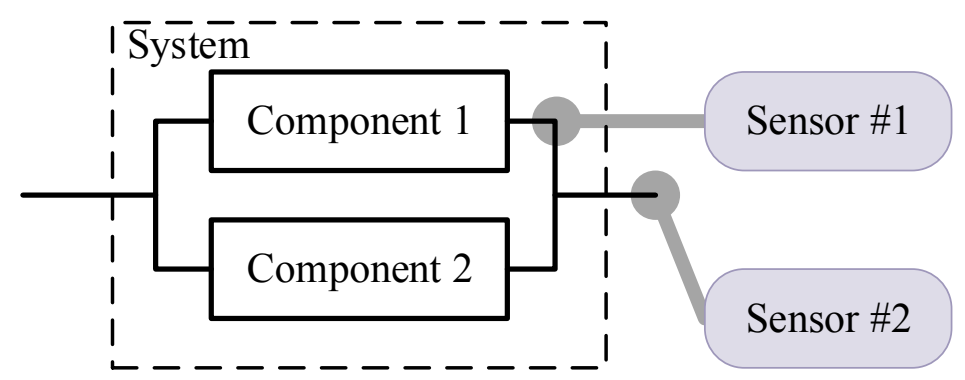

Fig. 1 Parallel system with two installed sensors

According to the Bayesian theorem, the parameter vector $\theta=\left(\lambda_{1}, \lambda_{2}\right)$ can be estimated as

$$
\pi(\boldsymbol{\theta} \mid D)=\frac{L(D \mid \boldsymbol{\theta}) \pi_{0}(\boldsymbol{\theta})}{\int L(D \mid \boldsymbol{\theta}) \pi_{0}(\boldsymbol{\theta}) d(\boldsymbol{\theta})}
$$

Given the prior distributions of $\boldsymbol{\theta}$, the key point here is how to formulate the likelihood function. 


\section{Scenario 1. Independent data}

This scenario is equivalent to that the system has been tested twice independently. The observation of "component 1 is failed" does not affect the likelihood of system failure as the data is collected at two isolated times.

The likelihood function is directly calculated by multiplying the two individual likelihoods as

$$
L\left(t_{1} \text { and } t_{S} \text { are independent data }\right)=f_{1}\left(t_{1} \mid \lambda_{1}\right)\left[f_{1}\left(t_{S} \mid \lambda_{1}\right) F_{2}\left(t_{S} \mid \lambda_{2}\right)+F_{1}\left(t_{S} \mid \lambda_{1}\right) f_{2}\left(t_{S} \mid \lambda_{2}\right)\right]
$$

where $f_{1}\left(t \mid \lambda_{1}\right), f_{2}\left(t \mid \lambda_{2}\right)$ denote the probability density functions (PDF) of time-to-failure for component 1 and component 2, and $F_{1}\left(t \mid \lambda_{1}\right), F_{2}\left(t \mid \lambda_{2}\right)$ denote the corresponding cumulative density functions (CDF).

\section{Scenario 2. Dependent data}

This scenario corresponds to the system being tested only once. It is observed that component 1 fails first at $t_{1}$ and the whole system fails subsequently at $t_{s}$. From the parallel system configuration, we can learn that component 2 fails at $t_{s}$. This is because the component 1 has already failed before and it is only the failure of both components that could lead to the failure of the whole system. In this manner, the likelihood should be constructed by considering the contributions from component 1 and component 2 . That is,

$$
L\left(t_{1} \text { and } t_{S} \text { are dependent data }\right)=f_{1}\left(t_{1} \mid \lambda_{1}\right) f_{2}\left(t_{S} \mid \lambda_{2}\right)
$$

It is obvious that the likelihood function derived in Eq. (3) has a primary difference compared with that in Eq. (2), suggesting the dependent data does not infer the same information when it is treated as independent data. When multi-level data becomes available, a comprehensive approach is required to correctly and efficiently aggregate the dependent information of a complex system for reliability analysis.

2.2 Bayesian methods for system reliability using multi-source information

\subsubsection{Independent information integration for multi-level system reliability models}


The Bayesian method is very appealing in the integration of multi-source information for reliability analysis.

Early works from Martz and Wailer [12] explored the system reliability when multi-level data is available. However, this study is only limited to simple system structures e.g. series/parallel system with binary data. Johnson [13] developed a hierarchical Bayesian model for early system reliability analysis when limited system-level data is available. Its novelty includes a unique "quality" index established to distinguish the system produced by “experienced” \& "inexperienced" manufactures. Hamada et al. [14] proposed a full Bayesian approach to combine multi-level independent (termed as non-overlapping by them) data in the quantification of a Fault Tree (FT). Grave et al. [15] followed this research line and extended the work to a multi-state system.

Recently, Straub [16], [17] established enhanced BNs for reliability and risk analysis of structural systems by combining traditional BNs and structural reliability methods. Guo et al. [18], [19] proposed a system reliability assessment approach taking multi-level information into account where special attention is paid on a scenario that multiple prior knowledge exists. Guo, Huang, and Peng [20] proposed a Bayesian information fusion approach to leverage the degradation information from multiple sources. Reese et al. [21] presented a Bayesian model to naturally integrate multi-source life information such as the life data obtained from the system, subsystem, component level, and the prior information in any level. Wilson et al. [22] demonstrated a practical example of reliability and uncertainty quantification via a combination of different types of data. Guo and Wilson [23] proposed a Bayesian approach for system evaluation using heterogeneous data sets. Li et al. [24]-[26] proposed a comprehensive Bayesian approach to aggregate heterogeneous data sets. Recent advances in system reliability with independent data combination are well summarized in [27], [28].

\subsubsection{Dependent information integration for multi-level system reliability models}

Addressing dependent information, Coolen [29] established the novel concept of "survival signature" for 
system reliability analysis. Following this research line, Patelli [30], [31] designed simulation algorithms for its implementation in the system reliability quantification. Aslett [32] presented its use from a Bayesian perspective. Walter et al. further extended it to a scenario that sets of prior exist [33] and prior-data conflict [34]. Focusing on the dependent data, Grave et al. [35] proposed a cut set-based method to incorporate the dependent data, in which a four-step algorithm is developed for implementation. Kim [36] established a practical method for mitigating the Bayesian anomaly. Jackson and Mosleh [37]-[40] discovered the role of dependent evidence played in the Bayesian inference and further developed comprehensive methodologies for on-demand systems [37], [38] as well as continuous life metric systems [39], [40]. Grave and Hamada [41] proposed a method to correctly construct the likelihood for simultaneous failure time data. Lin [42] proposed a copula-based approach addressing the formulation of a dependent structure. However, in previous studies, the system reliability models are usually limited to a RBD or a FT. As an alternative, BN has a more flexible capacity to represent a multi-level system structure. In the area dependence modeling using $\mathrm{BN}$, researchers at Memorial University have made a worthy contribution [43]. Khakzad and Khan [44] introduce the bow-tie (BT) analysis in dynamic safety analysis by representing the conditional dependence in the BN model [45], [46]. They employed copula functions to model the joint probability distributions of causations in the BT model of the accident scenario. The application fields include major accident modelling using spare data [47], rare event modelling considering dependence [48], process fault detection and diagnosis using BN [49] and Copula BN [50], and risk analysis or assessment to asset integrity [51] etc..

For the integration of dependent data in a BN model, Yontay and Pan [52] proposed a computational Bayesian approach to assess the dependence of a hierarchical system. The condition probabilities are estimated based on the combination of discrete data and expert opinions from multi-level. Further, Pan and Yontay [53] extended this study to a hierarchical system with continuous failure time data. However, since the study is only applied to a hierarchical system but not a general BN model, its applicability is limited to a system without sharing components. As a 
summary, none of the existing studies explicitly address the reliability of a general BN model with dependent data. In this paper, we focus on this unresolved issue and aim to develop a general Bayesian approach for a multi-level system with dependent evidence.

\section{Preliminaries}

From the topological perspective, a BN model is a Directed Acyclic Graph (DAG) which is composed of the nodes representing random variables, and the arcs representing the dependence among nodes. Given a BN model with a directed path from $N_{i}$ to $N_{j}$, the node $N_{i}$ is said to be the predecessor node of $N_{j}$, and conversely, $N_{j}$ is said to be the descendant node of $N_{i}$. In this paper, the predecessor nodes set of $N_{j}$ is denoted as $\mathbf{A}_{j}$. For any $N_{i} \in \mathbf{A}_{j}$, if $N_{i}$ has an arc directed into $N_{j}$, it is said to be a parent node (or direct predecessor) of $N_{j}$, and $N_{j}$ is the child node of $N_{i}$.

For a simple BN shown in Fig. 2, the predecessor nodes set of $N_{1}$ is $\mathbf{A}_{1}=\left(N_{2}, N_{3}, N_{4}\right)$ and the direct predecessor nodes set is $\mathbf{Q}_{1}=\left(N_{2}\right)$.

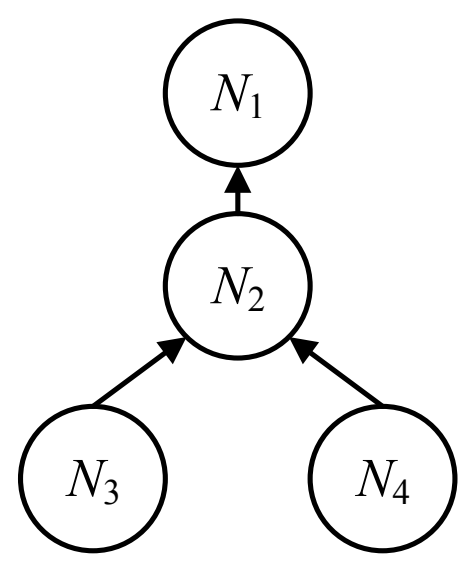

Fig. 2 A simple BN model

Any two nodes that are not directedly linked by an arc are said to be conditionally independent. One benefit of the $\mathrm{BN}$ is that the complex joint likelihood can be decomposed as a product of several conditional probabilities using the conditional independence. For instance, the probabilistic graphic model of the BN shown in Fig. 2 is represented as 


$$
\begin{aligned}
P\left(N_{1}, N_{2}, N_{3}, N_{4}\right) & =\prod_{i=1}^{4} P\left(N_{i} \mid Q_{i}\right) \\
& =P\left(N_{1} \mid N_{2}\right) P\left(N_{2} \mid N_{3}, N_{4}\right) P\left(N_{3}\right) P\left(N_{4}\right)
\end{aligned}
$$

where $P\left(N_{3}\right), P\left(N_{4}\right)$ are marginal probabilities and $P\left(N_{1} \mid N_{2}\right), P\left(N_{2} \mid N_{3}, N_{4}\right)$ are conditional probabilities. For the system reliability analysis, we also adopt the following conventions in the rest of this paper:

(i) the set of nodes without arcs directed into them (usually located at the bottom level) is denoted as component $\mathbf{C}$;

(ii) the set of nodes with arcs directed into it (usually located at the intermediate/top level) is denoted as system/subsystem $\mathbf{S}$;

(iii) the set of nodes with an observation (failure time data or censored data) is denoted as $\mathbf{O}$.

\section{Methodology}

The likelihood function plays a central role in Bayesian inference. For dependent data, the system likelihood function of a complex system has an encompassing form and cannot be constructed by simply multiplying each individual likelihood. In the proposed method, we calculate the overall likelihood function via a joint likelihood decomposition approach.

\subsection{A likelihood decomposition approach}

To illustrate the principle of our approach, consider a 3-level BN model shown in Fig. 3. In this paper, we consider a time-based system with binary states, namely, the state of a node can be either functioning (denoted as "1") or failed (denoted as "0"). The conditional probability table (CPT) parameters are given in Eq. (5) and the failure time $\left\{E_{0}: t=t_{0}, E_{1}: t=t_{1}, E_{2}: t=t_{2}\right\}$ of the three nodes $S_{0}, S_{1}$ and $C_{2}$ are observed. Compared with a typical hierarchical system, this $\mathrm{BN}$ model contains a sharing node $C_{2}$ which means the likelihood cannot be derived using traditional methods. 


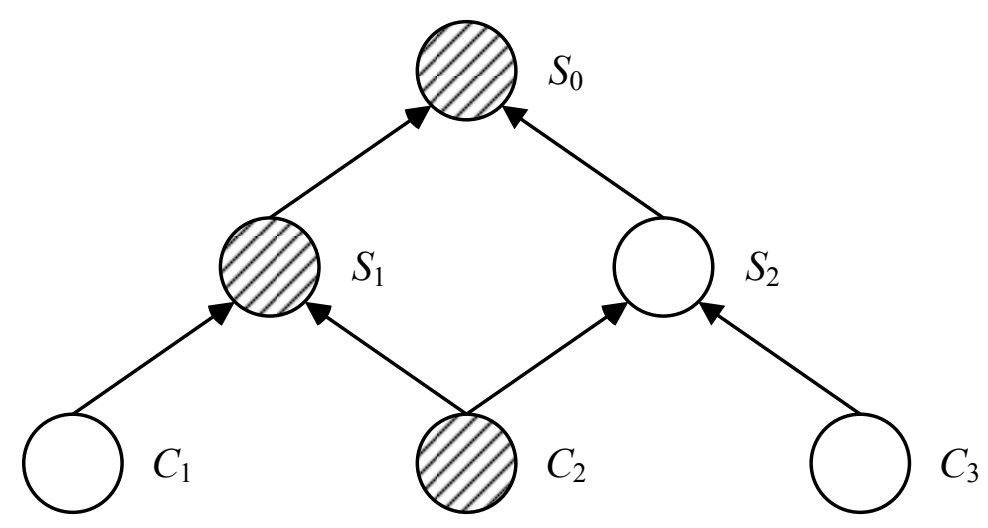

Fig. 3 3-level BN model

$\begin{array}{lll}\operatorname{Pr}\left(S_{0}=0 \mid S_{1}=0, S_{2}=0\right)=p_{000} & \operatorname{Pr}\left(S_{1}=0 \mid C_{1}=0, C_{2}=0\right)=p_{100} & \operatorname{Pr}\left(S_{2}=0 \mid C_{2}=0, C_{3}=0\right)=p_{200} \\ \operatorname{Pr}\left(S_{0}=0 \mid S_{1}=1, S_{2}=0\right)=p_{010} & \operatorname{Pr}\left(S_{1}=0 \mid C_{1}=1, C_{2}=0\right)=p_{110} & \operatorname{Pr}\left(S_{2}=0 \mid C_{2}=1, C_{3}=0\right)=p_{210} \\ \operatorname{Pr}\left(S_{0}=0 \mid S_{1}=0, S_{2}=1\right)=p_{001} & \operatorname{Pr}\left(S_{1}=0 \mid C_{1}=0, C_{2}=1\right)=p_{101} & \operatorname{Pr}\left(S_{2}=0 \mid C_{2}=0, C_{3}=1\right)=p_{201} \\ \operatorname{Pr}\left(S_{0}=0 \mid S_{1}=1, S_{2}=1\right)=p_{011} & \operatorname{Pr}\left(S_{1}=0 \mid C_{1}=1, C_{2}=1\right)=p_{111} & \operatorname{Pr}\left(S_{2}=0 \mid C_{2}=1, C_{3}=1\right)=p_{211}\end{array}$

From a mathematical perspective, the aim of our work is to represent the probability of observing these evidences (failure time $t_{0}, t_{1}$ and $t_{2}$ ) using the PDF or CDF of failure times of bottom-level components. In general, we have

$$
\begin{aligned}
F_{S_{i}}\left(t \mid \boldsymbol{\theta}_{S_{i}}\right) & =1-R_{S_{i}}\left(t \mid \boldsymbol{\theta}_{S_{i}}\right)=\Psi_{S_{i}}\left[\mathbf{p}, F_{S_{j}}\left(\boldsymbol{\theta}_{S_{j}}\right), F_{C_{j}}\left(\boldsymbol{\theta}_{S_{j}}\right): \forall S_{j} \in Q_{i}, \forall C_{j} \in Q_{i}\right] \\
f_{S_{i}}\left(t \mid \boldsymbol{\theta}_{S_{i}}\right) & =\frac{d F_{S_{i}}\left(t \mid \boldsymbol{\theta}_{S_{i}}\right)}{d t} \\
& =\sum_{\forall S_{j} \in \boldsymbol{Q}_{i}} \frac{\partial F_{S_{i}}(t)}{\partial F_{S_{j}}(t)} \times \frac{d F_{S_{j}}\left(t \mid \boldsymbol{\theta}_{S_{j}}\right)}{d t}+\sum_{\forall C_{j} \in \boldsymbol{Q}_{i}} \frac{\partial F_{S_{j}}(t)}{\partial F_{C_{j}}(t)} \times f_{C_{j}}\left(t \mid \boldsymbol{\theta}_{C_{j}}\right)
\end{aligned}
$$

where $\Psi$ is the system structure function determined by the system configuration; $\mathbf{p}$ is the CPT parameter vector; $\boldsymbol{\theta}$ is the distribution parameter vector; $S_{j}$ and $C_{j}$ denote the system/subsystem node and component node respectively; $\mathbf{Q}$ is the direct predecessor nodes set. Based on the system configuration, the overall joint likelihood function is decomposed as a product of three individual likelihoods

$$
L\left(E_{0}, E_{1}, E_{2}\right)=L_{1}\left(E_{0} \mid E_{1}, E_{2}\right) L\left(E_{1} \mid E_{2}\right) L\left(E_{2}\right)
$$

The individual likelihood $L\left(E_{2}\right)$ for the component $C_{2}$ is simply calculated as Eq. (8) since it is a bottom-level node without any lower level composing nodes.

$$
L\left(E_{2}: t=t_{2}\right)=\left.\frac{d F_{C_{2}}(t)}{d t}\right|_{t=t_{2}}=f_{C_{2}}\left(t_{2}\right)
$$


However, the derivation of likelihood functions for subsystem node $S_{1}$ and system node $S_{0}$ may require additional considerations. This is because the observed evidence $E_{2}$ may impact the formulation of $L\left(E_{0} \mid E_{1}, E_{2}\right)$ and $L\left(E_{1} \mid E_{2}\right)$ as they are conditioned on $E_{2}$. To better understand the likelihood formulation of a complex system in the presence of dependent data, we introduce the following notion of an inferential node.

Definition 1: For a system/subsystem node $S_{i}$, its predecessor node $N_{j} \in \mathbf{A}_{i} \quad$ ( $N_{j}$ could either be a subsystem node or a component node) is said to be inferential to $S_{i}$ if at least one propagating path from $N_{j}$ to $S_{i}: N_{\mathrm{j}} \rightarrow S_{\mathrm{i}}$ is active.

Examining the 3-level BN model shown in Fig. 4, it is found the state of $C_{1}$ will not affect the state of $S_{0}$ though $C_{1}$ is one predecessor of $S_{0}$. This is because the only propagating path $C_{1} \rightarrow S_{1} \rightarrow S_{0}$ is deactivated when an observation $t_{1}$ is available. Comparably, the state of component node $C_{2}$ will affect the state of system node $S_{0}$ even an observation for $S_{1}$ is available. This is because the information flow could bypass the blocked node $S_{1}$ and exert an influence on $S_{0}$ as the propagating path $C_{2} \rightarrow S_{2} \rightarrow S_{0}$ is activated.

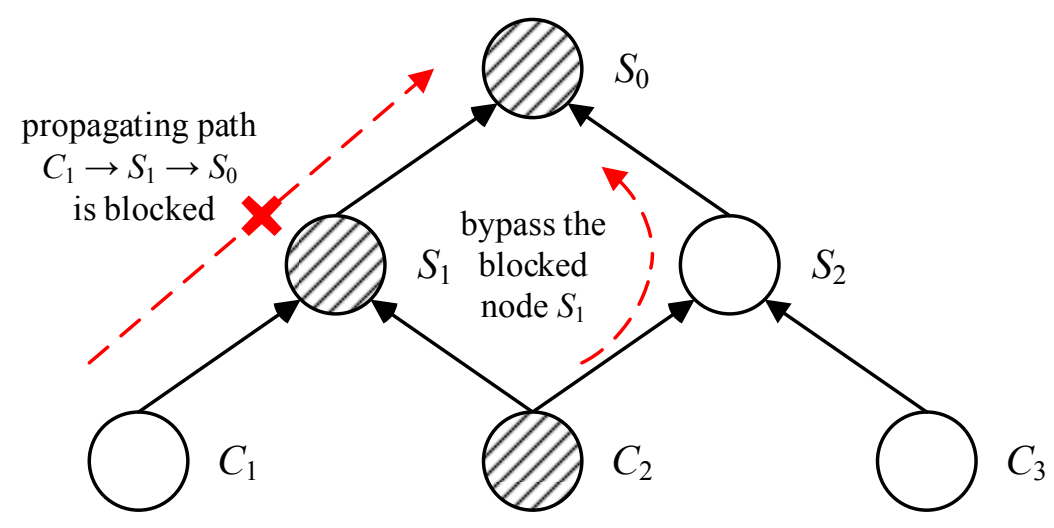

Fig. 4 Blocked propagating path and its bypass

Consequently, for the likelihood construction of any observed node in a system with multiple dependent data, only the influence of its inferential nodes should be taken into consideration. Using the advantages of the inferential node, the interactive relationship between any two nodes in a multi-level system is clearly revealed. 
4.2 Generating the explicit evidence and implicit evidence via an inferential diagram

By listing all inferential nodes of $S_{1}$ and $S_{0}, L\left(E_{1} \mid E_{2}\right)$ and $L\left(E_{0} \mid E_{1}, E_{2}\right)$ are given in form as

$$
\begin{aligned}
L\left(E_{1} \mid E_{2}\right)= & \frac{\partial F_{S_{1}}(t)}{\partial F_{C_{1}}(t)} \times f_{C_{1}}(t)+\frac{\partial F_{S_{1}}(t)}{\partial F_{C_{2}}(t)} \times f_{C_{2}}(t) \\
= & \left(p_{101}-p_{111}\right) f_{C_{1}}+\left(p_{110}-p_{111}\right) f_{C_{2}}+\left(p_{100}-p_{110}-p_{101}+p_{111}\right)\left(f_{C_{1}} F_{C_{2}}+F_{C_{1}} f_{C_{2}}\right) \\
L\left(E_{0} \mid E_{1}, E_{2}\right) & =\frac{\partial F_{S_{0}}(t)}{\partial F_{S_{1}}(t)} \times \frac{d F_{S_{1}}(t)}{d t}+\frac{\partial F_{S_{0}}(t)}{\partial F_{S_{2}}(t)} \times \frac{d F_{S_{2}}(t)}{d t} \\
& =\left(p_{001}-p_{011}\right) f_{S_{1}}+\left(p_{010}-p_{011}\right) f_{S_{2}}+\left(p_{000}-p_{010}-p_{001}+p_{011}\right)\left(f_{S_{1}} F_{S_{2}}+F_{S_{1}} f_{S_{2}}\right)
\end{aligned}
$$

To derive the explicit expressions of $L\left(E_{1} \mid E_{2}\right)$ and $L\left(E_{0} \mid E_{1}, E_{2}\right)$, collected evidence should be built into Eq.

(9) and (10). Given the available test data, uncertain PDFs or CDFs will collapse into a deterministic "jump" function. For instance, let $t_{2}<t_{1}<t_{0}$, the following three unit step functions are established.

$$
F_{S_{3}}(t)=H_{3}\left(t-t_{3}\right)=\left\{\begin{array}{ll}
0 & t<t_{3} \\
1 & t \geq t_{3}
\end{array}, \quad F_{S_{1}}(t)=H_{1}\left(t-t_{1}\right)=\left\{\begin{array}{ll}
0 & t<t_{1} \\
1 & t \geq t_{1}
\end{array}, \quad F_{S_{0}}(t)=H_{0}\left(t-t_{0}\right)= \begin{cases}0 & t<t_{0} \\
1 & t \geq t_{0}\end{cases}\right.\right.
$$

To investigate the implicit information embedded within the dependent evidence, we developed the following inferential diagram to generate necessary implicit evidence, which is consistent with both system configuration and the rationality of the presence of explicit evidence. The inferential diagram is developed based on the idea that by listing all explicit evidence in an ordinal sequence, the implicit evidence can be easily identified and compiled into the constructed likelihood functions. For example, besides the direct observation $t_{2}<t_{1}<t_{0}$, we can also learn that $F_{C_{2}}\left(t_{1}\right)=1, F_{C_{2}}\left(t_{0}\right)=1$, and $F_{S_{1}}\left(t_{0}\right)=1$ from the inferential diagram shown in Fig. 5 . Since these findings are not directly obtained from outer sources but are learned from the inference diagram taking into account of the system configuration, they are termed as implicit evidence. By contrast, failure time data or censored data that is directly obtained from an outer source, e.g. an installed sensor, is termed as explicit evidence. 


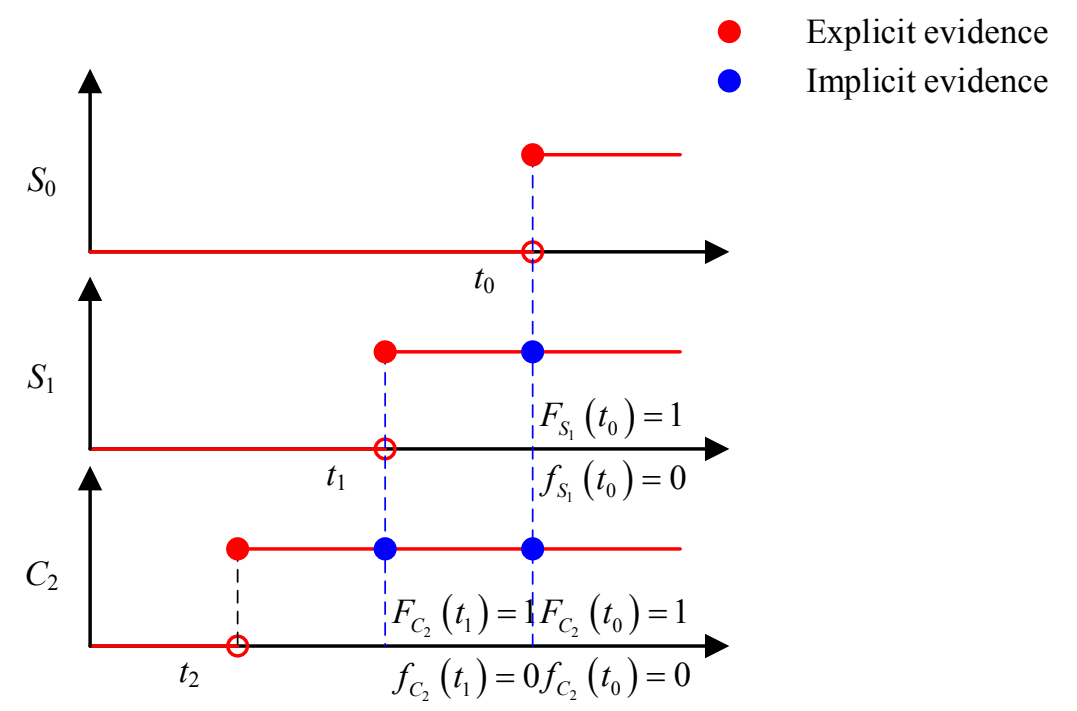

Fig. 5 Inferential diagram based on the explicit evidence $t_{2}<t_{1}<t_{0}$

To investigate the different roles of evidence in the likelihood construction, the following notions of Explicit Evidence-based Likelihood (EEL) and Implicit Evidence-based Likelihood (IEL) are introduced.

Definition 2. For a node $N_{i}$ with an observed failure time $t_{\mathrm{i}}$, If none of its inferential node has an explicit evidence i.e. $\boldsymbol{A}_{i} \cap \boldsymbol{O}=\varnothing$, its likelihood function $L_{i}$ is an Explicit Evidence-based Likelihood (EEL) function, otherwise, $L_{i}$ is an Implicit Evidence-based Likelihood (IEL) function. It follows, that

(i) If the likelihood of a node is an EEL, all its predecessors are inferential nodes.

(ii) The likelihood function for any observed component node is an EEL.

To determine the type of likelihood function of a subsystem or system-level node, we have to check if there is another observation available in the same branch of this network model. As presented in Eq. (8), the derivation of a EEL is straightforward by calculating the differential of its CDF of failure time.

$$
L\left(E_{i}\right)=\sum_{\substack{C_{j} \in \mathbf{A}_{i} \\ C_{j} \notin \mathbf{O}}} \frac{\partial F_{N_{i}}(t)}{\partial F_{C_{j}}(t)} \times f_{C_{j}}(t)
$$

Given the failure time data $t_{2}$, the likelihood function of component $C_{2}$ is calculated by substituting the evidence $E_{2}$ : $t=t_{2}$ into Eq. (8). However, for an IEL, not only the observed data but also the implicit evidence will exert influence on its the expression. The general form of an IEL could be expressed as 


$$
L\left(E_{j} \mid E_{k}\right)=\left\{\begin{array}{cc}
\sum_{\substack{N_{k} \in \mathbf{I}_{\mathbf{j}} \\
N_{k} \in \mathbf{O}}} \frac{\partial F_{N_{j}}(t)}{\partial F_{N_{k}}(t)} & \text { if } \exists t_{k}=t_{j} \\
\sum_{\substack{C_{i} \in \mathbf{I}_{i} \\
C_{l} \notin \mathbf{O}}} \frac{\partial F_{N_{j}}(t)}{\partial F_{C_{l}}(t)} \times f_{C_{l}}(t) & \text { otherwise }
\end{array}\right.
$$

Compared with the expression of EEL shown in Eq. (12), the IEL is composed of two parts. The first term in the right hand side represents the likelihood contributions from unobserved components and the second term represents the likelihood contributions from observed nodes. Given the failure probabilities between adjacent nodes

$$
\begin{aligned}
& F_{S_{0}}=p_{000} F_{S_{1}} F_{S_{2}}+p_{010}\left(1-F_{S_{1}}\right) F_{S_{2}}+p_{001} F_{S_{1}}\left(1-F_{S_{2}}\right)+p_{011}\left(1-F_{S_{1}}\right)\left(1-F_{S_{2}}\right) \\
& F_{S_{1}}=p_{100} F_{C_{1}} F_{C_{2}}+p_{110}\left(1-F_{C_{1}}\right) F_{C_{2}}+p_{101} F_{C_{1}}\left(1-F_{C_{2}}\right)+p_{111}\left(1-F_{C_{1}}\right)\left(1-F_{C_{2}}\right) \\
& F_{S_{2}}=p_{200} F_{C_{2}} F_{C_{3}}+p_{210}\left(1-F_{C_{2}}\right) F_{C_{3}}+p_{201} F_{C_{2}}\left(1-F_{C_{3}}\right)+p_{211}\left(1-F_{C_{2}}\right)\left(1-F_{C_{3}}\right)
\end{aligned}
$$

We have

$$
\begin{aligned}
& f_{S_{0}}=\left(p_{001}-p_{011}\right) f_{S_{1}}+\left(p_{010}-p_{011}\right) f_{S_{2}}+\left(p_{000}-p_{010}-p_{001}+p_{011}\right)\left(f_{S_{1}} F_{S_{2}}+F_{S_{1}} f_{S_{2}}\right) \\
& f_{S_{1}}=\left(p_{101}-p_{111}\right) f_{C_{1}}+\left(p_{110}-p_{111}\right) f_{C_{2}}+\left(p_{100}-p_{110}-p_{101}+p_{111}\right)\left(f_{C_{1}} F_{C_{2}}+F_{C_{1}} f_{C_{2}}\right) \\
& f_{S_{2}}=\left(p_{201}-p_{211}\right) f_{C_{2}}+\left(p_{210}-p_{211}\right) f_{C_{3}}+\left(p_{200}-p_{210}-p_{201}+p_{211}\right)\left(f_{C_{2}} F_{C_{3}}+F_{C_{2}} f_{C_{3}}\right)
\end{aligned}
$$

the two IEL functions for $L\left(E_{1} \mid E_{2}\right)$ and $L\left(E_{0} \mid E_{1}, E_{2}\right)$ are calculated by plugging Eq. (14), (15) and the generated implicit evidence $F_{C_{2}}\left(t_{1}\right)=1, F_{C_{2}}\left(t_{0}\right)=1$, and $F_{S_{1}}\left(t_{0}\right)=1$ into Eq. (13)

$$
\begin{aligned}
& \begin{aligned}
L\left(E_{1}: t=t_{1} \mid E_{2}: t=t_{2}\right) & =\left.\frac{\partial F_{S_{1}}(t)}{\partial F_{C_{1}}(t)}\right|_{t=t_{1}} \times\left. f_{C_{1}}(t)\right|_{t=t_{1}} \\
& =\left(p_{101}-p_{111}\right) f_{C_{1}}\left(t_{1}\right)
\end{aligned} \\
& \begin{aligned}
L\left(E_{0}: t=t_{0} \mid E_{1}: t=t_{1}, E_{2}: t=t_{2}\right) & =\left.\frac{\partial F_{S_{0}}(t)}{\partial F_{C_{3}}(t)}\right|_{t=t_{0}} \times\left. f_{C_{3}}\right|_{t=t_{0}} \\
& =\left(p_{000}-p_{001}\right)\left(p_{200}-p_{201}\right) f_{C_{3}}\left(t_{0}\right)
\end{aligned}
\end{aligned}
$$

Note that the likelihood contribution from $C_{2}$ has been derived in the EEL, so here it only serves as a piece of evidence. 
4.3 The role of implicit evidence in the likelihood construction

For a EEL function, the value of an observation (explicit evidence) will not affect its expression form. That is, once the system configuration is fully specified, the expression of an EEL is settled. However, for the formulation of an IEL function, the (both explicit and implicit) evidence has a more complicated impact. To demonstrate this point, we modify the explicit evidence as $t_{2}>t_{1}>t_{0}$ (which indicates that the system $S_{0}$ is failed before the subsystem $S_{1}$ and subsequently the component $C_{2}$ ), then the corresponding inferential diagram is drawn as Fig. 6.

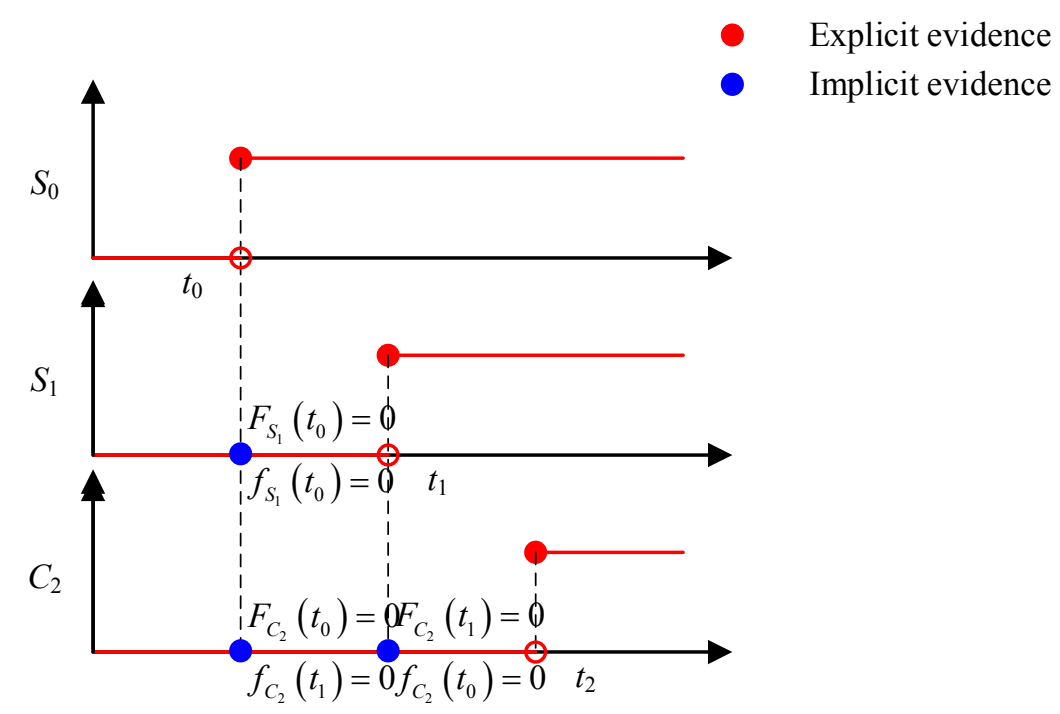

Fig. 6 Inferential diagram based on the explicit evidence $t_{2}>t_{1}>t_{0}$

By substituting the implicit evidence $F_{C_{2}}\left(t_{1}\right)=0, F_{C_{2}}\left(t_{0}\right)=0$, and $F_{S_{1}}\left(t_{0}\right)=0$ into Eq. (13), the two IEL functions are now derived in the following Eq. (18) and (19)

$$
\begin{aligned}
& \begin{aligned}
L\left(E_{1}: t=t_{1} \mid E_{2}: t=t_{2}\right) & =\left.\frac{\partial F_{S_{1}}(t)}{\partial F_{C_{1}}(t)}\right|_{t=t_{1}} \times\left. f_{C_{1}}(t)\right|_{t=t_{1}} \\
& =\left(p_{110}-p_{100}\right) f_{C_{1}}\left(t_{1}\right)
\end{aligned} \\
& \begin{aligned}
L\left(E_{0}: t=t_{0} \mid E_{1}: t=t_{1}, E_{2}: t=t_{2}\right) & =\left.\frac{\partial F_{S_{0}}(t)}{\partial F_{C_{3}}(t)}\right|_{t=t_{0}} \times\left. f_{C_{3}}\right|_{t=t_{0}} \\
& =\left(p_{010}-p_{011}\right)\left(p_{210}-p_{211}\right) f_{C_{3}}\left(t_{0}\right)
\end{aligned}
\end{aligned}
$$

It is found that the IEL functions derived in Eq. (18) and (19) are substantially different from the IEL function in Eq. (16) and (17) as their expressions contain different CPT parameters. Compared with the explicit evidence, 
the implicit evidence will affect the expression of an IEL function as it is developed through an inference taking system configuration into consideration.

4.4 Implementation - a full Bayesian approach for system reliability analysis in the presence of dependent data

For the practical engineering use, we present the proposed full Bayesian approach in the following Fig. 7 and illustrate its implementation procedure step by step.

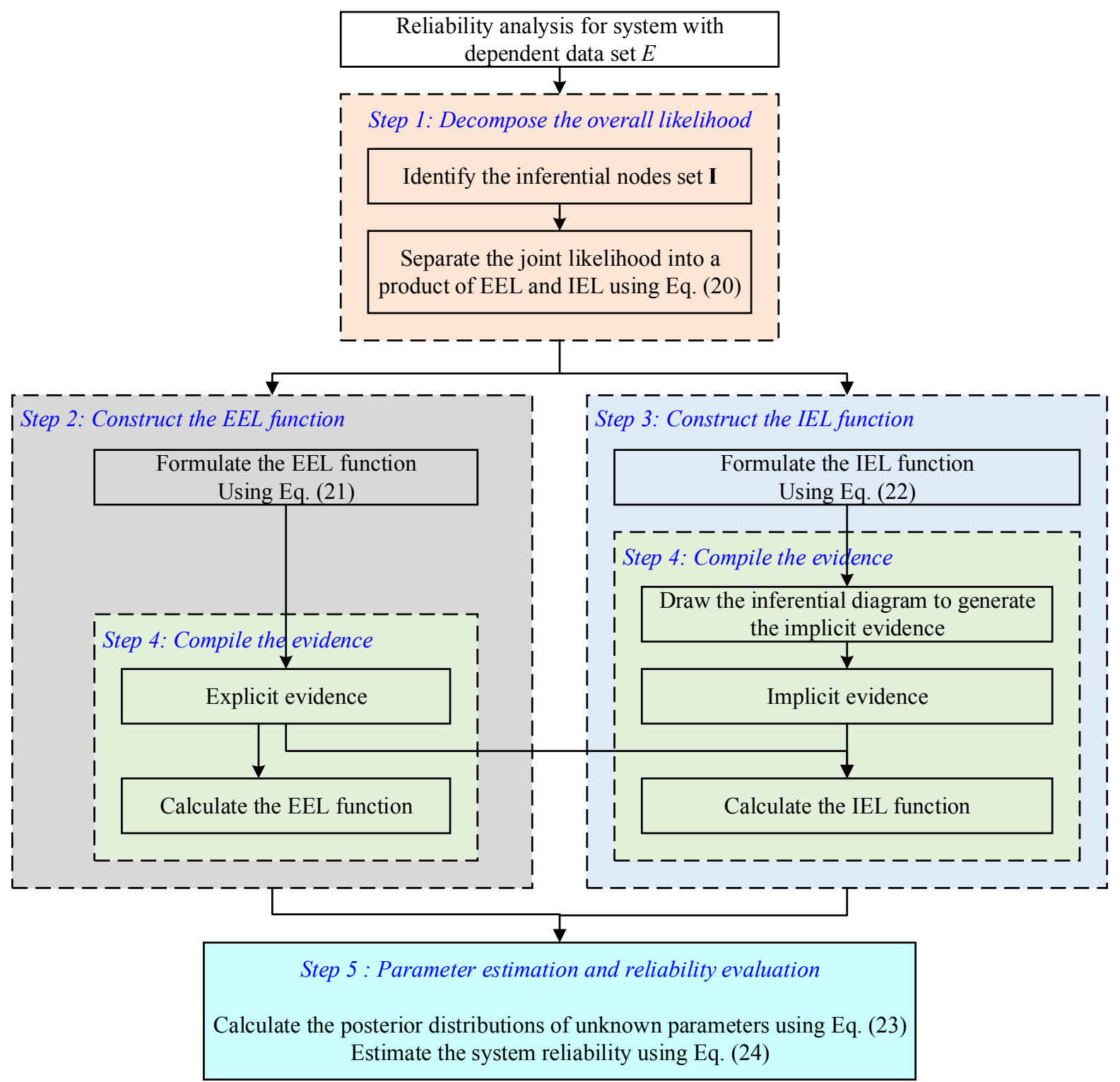

Fig. 7 Schematic of the proposed method for system reliability analysis with dependent data. 
Step 1 Decompose the overall likelihood

Identify the inferential nodes set $\mathbf{I}$ for all nodes with explicit evidence and then decompose the overall likelihood into the product of several EEL functions and IEL functions as Eq. (20).

$$
L\left(E_{1}, E_{i}, \ldots, E_{n}\right)=\prod_{N_{i} \in \mathbf{O}, \mathbf{A}_{i} \cap \mathbf{O}=\varnothing}^{\mathbf{U}} L^{E E L}\left(E_{i}\right) \prod_{\substack{N_{j} \in \mathbf{O}, \mathbf{A}_{j} \cap \mathbf{O}=\varnothing \\ N_{k} \in \mathbf{O}, N_{k} \in \mathbf{I}_{j}}}^{\mathbf{v}} L^{I E L}\left(E_{j} \mid E_{k}\right)
$$

where $\mathbf{U}: \forall N_{i} \in \mathbf{O}, \mathbf{A}_{i} \cap \mathbf{O}=\varnothing$ is the set of nodes that the likelihood of which is EEL, $\quad \mathbf{V}: \forall V_{j} \in \mathbf{O}, \mathbf{A}_{j} \cap \mathbf{O} \neq \varnothing$ is the set of nodes that the likelihood of which is IEL, $\mathbf{I}_{j}$ is the set of inferential nodes of $N_{j}$.

The node with an EEL function could be either a component node or a system/subsystem node but the node with an IEL function must be a system/subsystem node. There are as many EEL functions and IEL functions as the number of nodes with explicit evidence.

\section{Step 2 Construct the EEL function}

Formulate the EEL function using the following Eq. (21)

$$
L^{E E L}\left(E_{i}\right)=\sum_{\substack{C_{j} \in \mathbf{A}_{i} \\ C_{j} \notin \mathbf{O}}} \frac{\partial F_{N_{i}}(t)}{\partial F_{C_{j}}(t)} \times f_{C_{j}}(t)
$$

where $\mathbf{A}$ is the predecessor nodes set, $\mathbf{O}$ is the observed nodes set, and Eq. (6) should be taken into account to simplify the expression of an EEL function.

\section{Step 3 Construct the IEL function}

Formulate the IEL function using the following Eq. (22)

$$
L^{I E L}\left(E_{j} \mid E_{k}\right)=\left\{\begin{array}{cc}
\sum_{\substack{N_{k} \in \mathbf{I}_{i} \\
N_{k} \in \mathbf{O}}} \frac{\partial F_{N_{j}}(t)}{\partial F_{N_{k}}(t)} & \exists E_{k}=E_{j} \\
\sum_{\substack{C_{l} \in \mathbf{I}_{j} \\
C_{l} \notin \mathbf{O}}} \frac{\partial F_{N_{j}}(t)}{\partial F_{C_{l}}(t)} \times f_{C_{l}}(t) & \text { otherwise }
\end{array}\right.
$$

where $\mathbf{I}$ is the inferential nodes set, $\mathbf{O}$ is the observed nodes set, and Eq. (6) should be taken into account to simplify the expression of an IEL function. 
Step 4 Generate the implicit evidence and compile the evidence to calculate all EELs and IELs

Draw the inferential diagram by listing all explicit evidence in a time sequence and generate the implicit evidence from the corresponding unit step function. For an EEL function, compile explicit evidence into Eq. (21) to simplify its expression. To calculate the IEL function, both explicit evidence and implicit evidence should be considered. The IEL function constructed in Eq. (22) should be represented as a function of known PDFs or CDFs of time-to-failure of inferentially nodes or components. Note that here we use the term "evidence" instead of "data" because this notion covers a more wide range of available information such as an observed time-to-failure (failure time data) or a knowledge that the system is not failed until a specific time (censored data). In this situation, The contributions of an EEL function or an IEL function could be either the probability density for an exact failure time data or a probability for the censored data.

\section{Step 5 Parameter estimation and reliability evaluation}

The overall likelihood is constructed by multiplying all EEL functions and IEL functions. Once the likelihood function is fully determined, the posterior distribution of unknown parameters (both model parameters and distribution parameters) are estimated using the Bayesian rule as Eq. (23).

$$
\pi(\boldsymbol{\theta}, \mathbf{p} \mid E)=\frac{L(E \mid \boldsymbol{\theta}, \mathbf{p}) \pi_{0}(\boldsymbol{\theta}, \mathbf{p})}{\int_{\forall \boldsymbol{\theta}, \forall \mathbf{p}} L(E \mid \boldsymbol{\theta}, \mathbf{p}) \pi_{0}(\boldsymbol{\theta}, \mathbf{p}) d(\boldsymbol{\theta}, \mathbf{p})}
$$

The system reliability that is of interest is estimated accordingly.

$$
R_{S_{i}}\left(t \mid \boldsymbol{\theta}_{S_{i}}\right)=1-\Psi_{S_{i}}\left[\mathbf{p}, F_{S_{j}}\left(\boldsymbol{\theta}_{S_{j}}\right), F_{C_{j}}\left(\boldsymbol{\theta}_{S_{j}}\right): \forall S_{j} \in Q_{i}, \forall C_{j} \in Q_{i}\right]
$$

Since the proposed reliability method has no specific requirement on the model structure, it allows an extensive application field including both hierarchical systems and BN models with hybrid structures. However, it should be noted that above development of this approach is under a static Bayesian framework. Extensions to a dynamic BN with time-dependent structure and/or evidence is possible but much efforts are required in the construction of inferential diagram since it varies with respect to both model structure and available information in such case. 


\section{Comparisons and validations}

In this section, we present three numerical case studies to demonstrate the proposed approach. Comparisons with well-established methods are conducted for validation purposes.

\subsection{Comparison with Jackson and Mosleh's method}

In Jackson and Mosleh's research [39], they proposed a Bayesian approach for system reliability evaluation addressing simultaneously collected test data.

Example 2 Jackson and Mosleh's case in [39]

Jackson and Mosleh presented a fault tree model with 3 sensors and 6 components as shown in Fig. 8. The available information includes the sensor detected time-to-failure $E=\left\{E_{1}, E_{2}, E_{3}\right\}=\left\{t_{1}^{S}=113.54, t_{2}^{S}>t_{2}^{*}=113.54, t_{3}^{S}=78.69\right\}$ and the PDFs of time-to-failure of the 6 components. We validate our method by calculating the system likelihood function and comparing the derived results with those presented in [39].

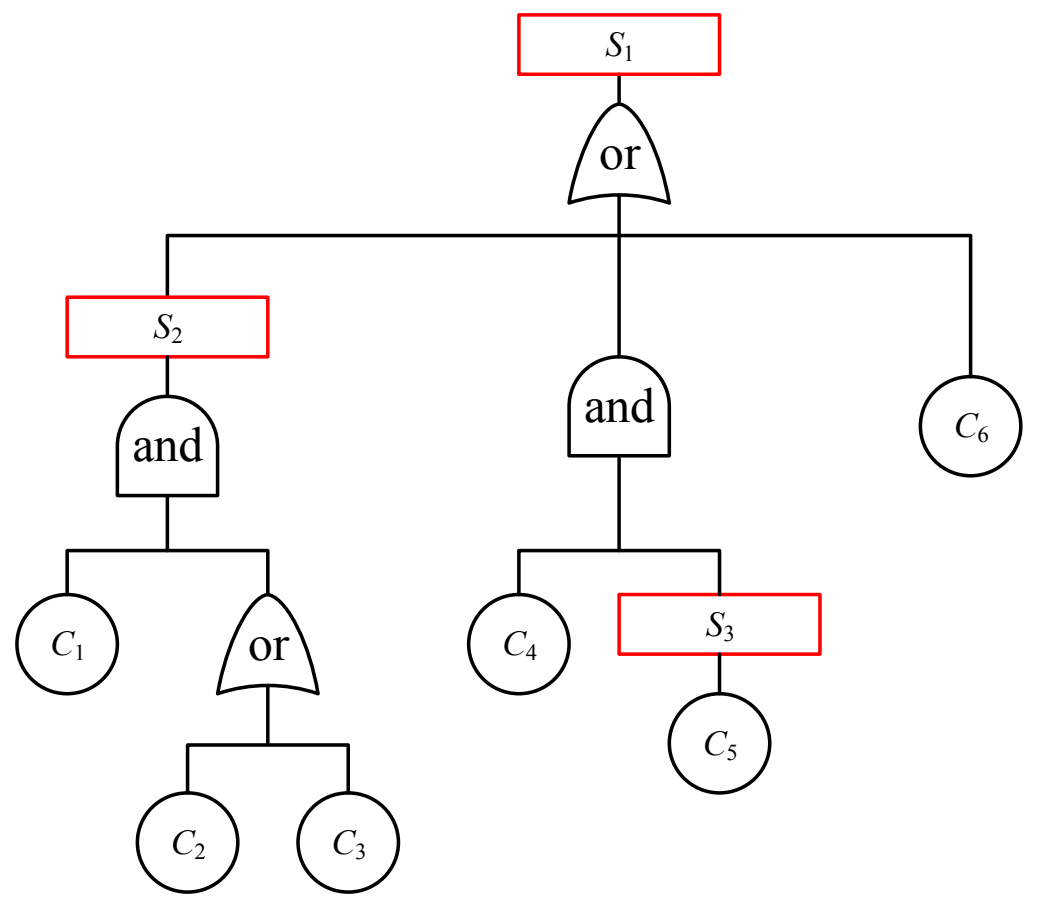

Fig. 8 Jackson and Mosleh's example in [39] 
Step 1 The overall likelihood is decomposed as the product of EEL functions and IEL functions in Eq. (25).

$$
L\left(E_{1}, E_{2}, E_{3}\right)=L\left(E_{1} \mid E_{2}, E_{3}\right) L\left(E_{2}\right) L\left(E_{3}\right)
$$

where the EEL node set $\mathbf{U}: S_{2}, S_{3}$ and the IEL node set $\mathbf{V}: S_{1}$.

Step 2 Using Eq. (21), the two EEL functions are derived as

$$
L\left(E_{2}\right)=1-F_{S_{2}} \quad L\left(E_{3}\right)=\frac{\partial F_{S_{3}}(t)}{\partial F_{C_{5}}(t)} \times f_{C_{5}}(t)=f_{5}
$$

Step 3 Using Eq. (22), the IEL function is derived as

$$
\begin{aligned}
L\left(E_{1} \mid E_{2}, E_{3}\right) & =\sum_{j=4,6} \frac{\partial F_{S_{1}}(t)}{\partial F_{C_{j}}(t)} \times f_{C_{j}}(t) \\
& =\left[\left(F_{3}^{S}-F_{2}^{S} F_{3}^{S}-F_{3}^{S} F_{6}+F_{2}^{S} F_{3}^{S} F_{6}\right) f_{4}+\left(1-F_{2}^{S}-F_{4} F_{3}^{S}+F_{2}^{S} F_{4} F_{3}^{S}\right) f_{6}\right]
\end{aligned}
$$

Step 3 Draw the inferential diagram shown in Fig. 9 to generate the implicit evidence presented in Eq. (28).

$$
F_{2}^{S}\left(t_{1}^{S}\right)=0, f_{2}^{S}\left(t_{1}^{S}\right)=0, F_{3}^{S}\left(t_{1}^{S}\right)=1, f_{3}^{S}\left(t_{1}^{S}\right)=0
$$

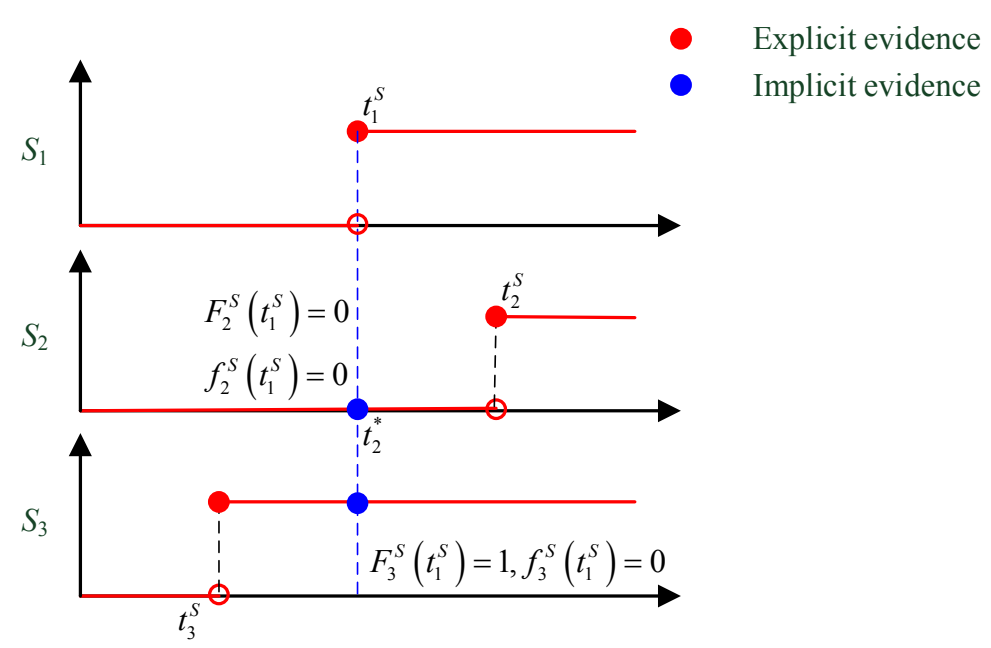

Fig. 9 Inferential diagram for example 2

After compiling the explicit evidence into Eq.(26), the EEL functions are calculated as

$$
L\left(E_{2}: t_{2}^{S}>t_{2}^{*}=113.54\right)=\left.\left[1-\left(F_{2}+F_{3}-F_{2} F_{3}\right) f_{1}\right]\right|_{t=t_{2}^{*}} \quad L\left(E_{3}: t_{3}^{S}=78.69\right)=\left.f_{5}\right|_{t=t_{3}^{S}}
$$

By substituting both explicit evidence and implicit evidence into Eq. (27), the IEL function is calculated as

$$
L\left(E_{1}: t_{1}^{S}=113.54 \mid E_{2}: t_{2}^{S}>t_{2}^{*}=113.54, E_{3}: t_{3}^{S}=78.69\right)=\left.\left[\left(1-F_{6}\right) f_{4}+\left(1-F_{4}\right) f_{6}\right]\right|_{t=t_{1}^{S}}
$$

Step 5 The overall likelihood is constructed by multiplying each individual EEL function and IEL function as 


$$
L\left(E_{1}, E_{2}, E_{3}\right)=\left.\left[\left(1-F_{6}\right) f_{4}+\left(1-F_{4}\right) f_{6}\right]\right|_{t=t_{1}^{s}} \times\left[1-\left(F_{2}+F_{3}-F_{2} F_{3}\right) f_{1}\right]_{t=t_{2}^{*}} \times\left. f_{5}\right|_{t=t_{3}}
$$

This result is equivalent to that presented by Jackson and Mosleh in [39].

\subsection{Comparison with Graves and Hamada's method}

Graves and Hamada [41] presented a method to evaluate the likelihood when simultaneous failure data appears.

Their method is performed by listing of all possible events consistent with the system configuration. We validate our proposed work by revisiting their case study examples.

Example 3 Graves and Hamada's case in [41]

Fig. 10 shows an event tree model of a prototypical system, where "O" stands for an "OR" gate; "A" stands for an "AND" gate. Given the simultaneously collected data $E=\left\{E_{1}, E_{2}, E_{3}\right\}=\left\{E_{1}: t_{S_{0}}=100, E_{2}: t_{S_{01}}=100, E_{3}: t_{S_{012}}>t_{S_{012}}^{*}=100\right\}$, we use the proposed method to derive its likelihood function.

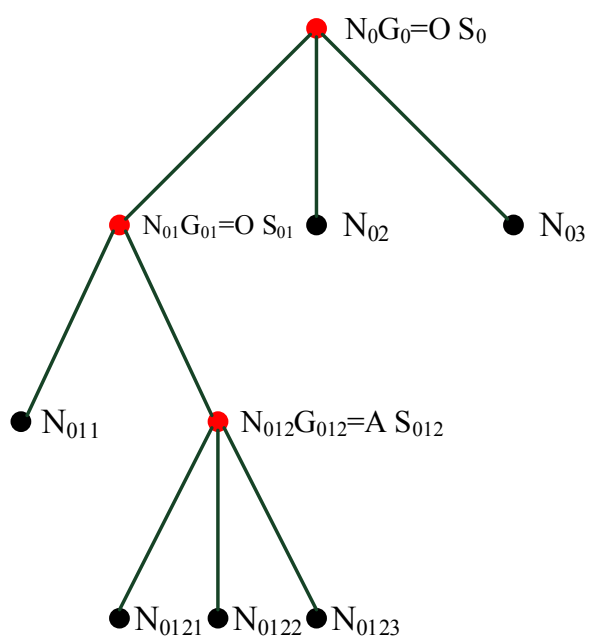

Fig. 10 Jackson and Mosleh's example in [41]

Step 1 The system likelihood is decomposed as the product of EEL functions and IEL functions as Eq. (32)

$$
L\left(E_{1}, E_{2}, E_{3}\right)=L\left(E_{3}\right) L\left(E_{2} \mid E_{3}\right) L\left(E_{1} \mid E_{2}\right)
$$

where the EEL node set $\mathbf{U}: S_{0}$ and the IEL node set $\mathbf{V}: S_{01}, S_{0}$. 
Step 2 Using Eq. (21), the EEL function is expressed as

$$
L\left(E_{3}\right)=1-F_{012}
$$

Step 3 Using Eq. (22), the two IEL functions are expressed as

$$
\begin{gathered}
L\left(E_{2} \mid E_{3}\right)=\frac{\partial F_{S_{01}}(t)}{\partial F_{N_{011}}(t)} \times f_{N_{011}}(t)=\left(1-F_{012}\right) f_{011} \\
L\left(E_{1} \mid E_{2}\right)=\frac{\partial F_{S_{0}}(t)}{\partial F_{S_{01}}(t)}=\left(1-F_{02}\right)\left(1-F_{03}\right)
\end{gathered}
$$

Step 4 Draw the inferential diagram shown in Fig. 11 to generate the implicit evidence presented in Eq. (36).

$$
F_{01}\left(t_{S_{0}}\right)=1, f_{01}\left(t_{S_{0}}\right)=1, F_{012}\left(t_{S_{01}}\right)=0, f_{012}\left(t_{S_{01}}\right)=0
$$

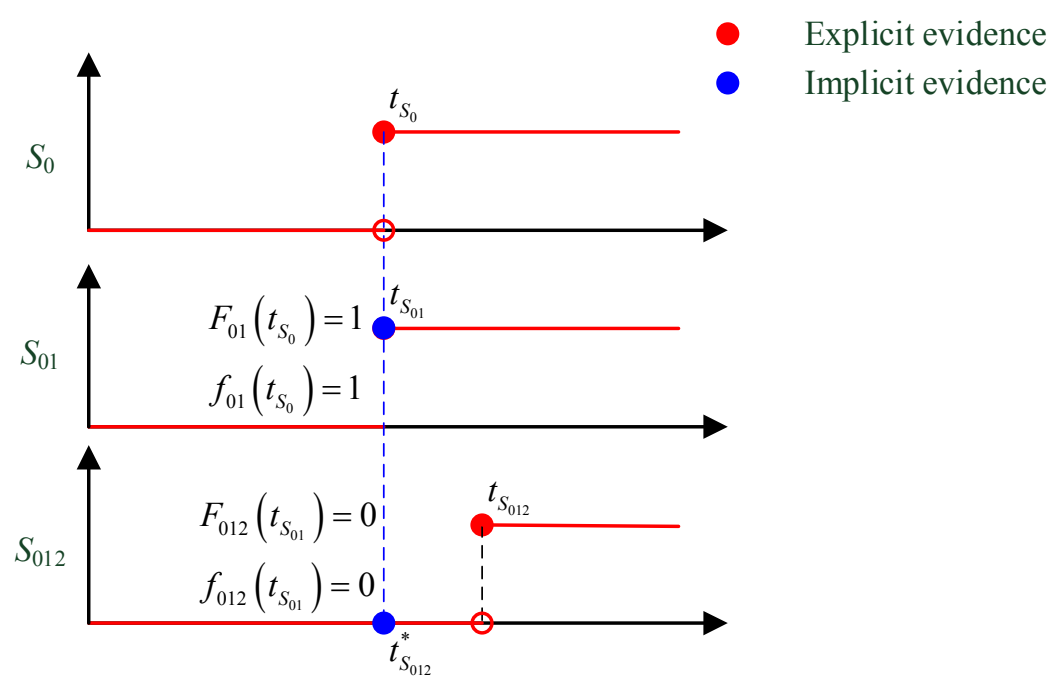

Fig. 11 Inferential diagram for example 3

After compiling the explicit evidence into Eq. (33), the EEL function is calculated as

$$
L\left(E_{3}: t_{S_{012}}>t_{S_{012}}^{*}=100\right)=1-\left.F_{0121} F_{0122} F_{0123}\right|_{t=t_{012}^{*}}
$$

By substituting both explicit evidence and implicit evidence into Eq. (34) and Eq. (35), two IEL functions are calculated as

$$
\begin{aligned}
& L\left(E_{2}: t_{S_{01}}=100 \mid E_{3}: t_{S_{012}}>t_{S_{012}}^{*}=100\right)=\left.f_{011}\right|_{t=t_{S_{01}}} \\
& L\left(E_{1}: t_{S_{0}}=100 \mid E_{2}: t_{S_{01}}=100\right)=\left.\left(1-F_{02}\right)\left(1-F_{03}\right)\right|_{t=t_{S_{0}}}
\end{aligned}
$$

Step 5 The overall likelihood is constructed by multiplying each individual EEL and IEL as 


$$
L\left(E_{1}, E_{2}, E_{3}\right)=\left.\left[\left(1-F_{02}\right)\left(1-F_{03}\right)\right]\right|_{t=t_{S_{0}}} \times\left. f_{011}\right|_{t=t_{t_{01}}} \times\left.\left(1-F_{0121} F_{0122} F_{0123}\right)\right|_{t=t_{S_{012}}^{*}}
$$

This result is equivalent to that presented by Graves and Hamada in [41]

\subsection{Comparison with Pan and Yontay’s method}

Pan and Yontay [53] developed a $d$-separation-based approach for the multi-level system using incomplete data. Their approach is efficient in reliability prediction for hierarchical BN models. We address their case by our method.

\section{Example 4 Pan and Yontay's case in [53]}

Fig. 12 shows a hierarchical BN model, which could be considered as an extension of the traditional FTA and ET.

Given the observed data $E=\left\{E_{1}: t_{C_{5}}=16, E_{2}: t_{S_{1}}=51, E_{3}: t_{S_{0}}<t_{t_{S_{0}}}^{*}=80\right\}$ and other basic pre-settings (refer [53] for details), the task here is to construct the system likelihood function.

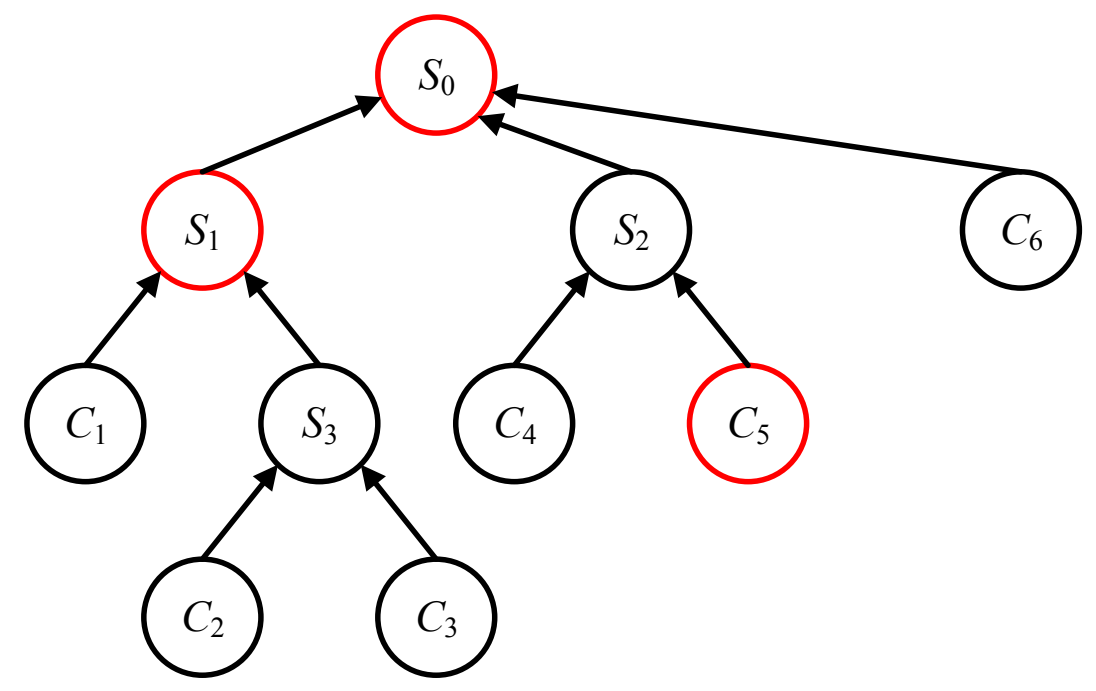

Fig. 12 Pan and Yontay's example

Step 1 The system likelihood is decomposed as the product of EEL functions and IEL functions in Eq. (41).

$$
L\left(E_{1}, E_{2}, E_{3}\right)=L\left(E_{1}\right) L\left(E_{2}\right) L\left(E_{3} \mid E_{1}, E_{2}\right)
$$

where the EEL node set $\mathbf{U}: C_{5}, S_{1}$, and the IEL node set $\mathbf{V}: S_{0}$.

Step 2 Using Eq. (21), the EEL functions are expressed as 


$$
\begin{gathered}
L\left(E_{1}\right)=\frac{\partial F_{C_{5}}(t)}{\partial F_{C_{5}}(t)} \times f_{C_{5}}(t)=f_{C_{5}} \\
L\left(E_{2}\right)=\sum_{j=1,2,3} \frac{\partial F_{S_{1}}(t)}{\partial F_{C_{j}}(t)} \times f_{C_{j}}(t) \\
=\left[F_{C_{2}}+F_{C_{3}}-F_{C_{2}} F_{C_{3}}\right] f_{C_{1}}+\left[F_{C_{1}}\left(1-F_{C_{3}}\right)\right] f_{C_{2}}+\left[F_{C_{1}}\left(1-F_{C_{2}}\right)\right] f_{C_{3}}
\end{gathered}
$$

Step 3 Using Eq. (22), the IEL is expressed as

$$
L\left(E_{3} \mid E_{1}, E_{2}\right)=F_{S_{0}}(t)=\left(\begin{array}{l}
p_{111} F_{S_{1}} F_{C_{4}} F_{C_{5}} F_{C_{6}}+p_{011}\left(1-F_{S_{1}}\right) F_{C_{4}} F_{C_{5}} F_{C_{6}} \\
+p_{101} F_{S_{1}}\left(1-F_{C_{4}} F_{C_{5}}\right) F_{C_{6}}+p_{110} F_{S_{1}} F_{C_{4}} F_{C_{5}}\left(1-F_{C_{6}}\right) \\
+p_{001}\left(1-F_{S_{1}}\right)\left(1-F_{C_{4}} F_{C_{5}}\right) F_{C_{6}}+p_{100} F_{S_{1}}\left(1-F_{C_{4}} F_{C_{5}}\right)\left(1-F_{C_{6}}\right) \\
+p_{010}\left(1-F_{S_{1}}\right) F_{C_{4}} F_{C_{5}}\left(1-F_{C_{6}}\right)+p_{110}\left(1-F_{S_{1}}\right)\left(1-F_{C_{4}} F_{C_{5}}\right)\left(1-F_{C_{6}}\right)
\end{array}\right)
$$

Step 4 Draw the inferential diagram shown in Fig. 13 to generate the implicit evidence presented in Eq. (45).

$$
F_{S_{1}}\left(t_{S_{0}}^{*}\right)=1, f_{S_{1}}\left(t_{S_{0}}^{*}\right)=0, F_{C_{5}}\left(t_{S_{0}}^{*}\right)=1, f_{C_{5}}\left(t_{S_{0}}^{*}\right)=0
$$

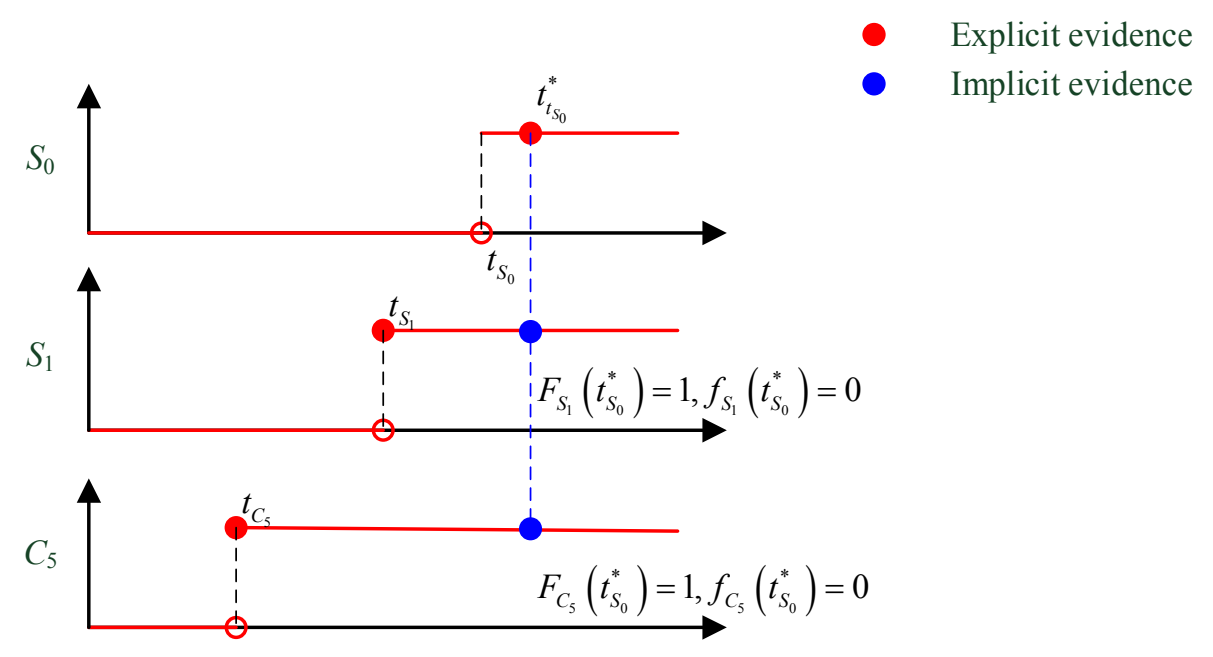

Fig. 13 Inferential diagram for example 4

After compiling the explicit evidence into Eq. (42) and (43), EEL functions are calculated as

$$
\begin{gathered}
L\left(E_{1}: t_{C_{5}}=16 ;\right)=\left.f_{C_{5}}\right|_{t=t_{C_{5}}} \\
L\left(E_{2}: t_{S_{1}}=51\right)=\left.\left[\left(F_{C_{2}}+F_{C_{3}}-F_{C_{2}} F_{C_{3}}\right) f_{C_{1}}+\left(F_{C_{1}}-F_{C_{1}} F_{C_{3}}\right) f_{C_{2}}+\left(F_{C_{1}}-F_{C_{1}} F_{C_{2}}\right) f_{C_{3}}\right]\right|_{t=t_{S_{1}}}
\end{gathered}
$$

By substituting both explicit evidence and implicit evidence into Eq. (44), the IEL is calculated as

$$
L\left(E_{3}: t_{S_{0}}<t_{S_{0}}^{*}=80 \mid E_{1}: t_{C_{5}}=16, E_{2}: t_{S_{1}}=51 ;\right)=\left.\left[\begin{array}{l}
p_{111} F_{C_{4}} F_{C_{6}}+p_{101}\left(1-F_{C_{4}}\right) F_{C_{6}} \\
+p_{110} F_{C_{4}}\left(1-F_{C_{6}}\right)+p_{100}\left(1-F_{C_{4}}\right)\left(1-F_{C_{6}}\right)
\end{array}\right]\right|_{t=t_{S_{0}}} ^{*}
$$


Step 5 The overall likelihood is constructed by multiplying each individual EEL and IEL function as

$$
L\left(E_{1}, E_{2}, E_{3}\right)=\left.f_{C_{5}}\right|_{t=t_{C_{5}}} \times\left[\begin{array}{l}
\left(F_{C_{2}}+F_{C_{3}}-F_{C_{2}} F_{C_{3}}\right) f_{C_{1}} \\
+\left(F_{C_{1}}\left(1-F_{C_{3}}\right)\right) f_{C_{2}} \\
+\left(F_{C_{1}}\left(1-F_{C_{2}}\right)\right) f_{C_{3}}
\end{array}\right] t_{t=t_{S_{1}}} \times\left.\left[\begin{array}{l}
p_{111} F_{C_{4}} F_{C_{6}}+p_{101}\left(1-F_{C_{4}}\right) F_{C_{6}} \\
+p_{110} F_{C_{4}}\left(1-F_{C_{6}}\right) \\
+p_{100}\left(1-F_{C_{4}}\right)\left(1-F_{C_{6}}\right)
\end{array}\right]\right|_{t=t_{S_{0}}}
$$

The result is equivalent to that presented by Pan and Yontay in [53].

\section{Application example}

In this section, We carry out a practical application example in which the problem is difficult to be solved by existing methods.

6.1 Model description and parameter settings

Example 5 Fig. 14 shows the system configuration of a Harmonic Gear Drive (HGD) device, which is widely used in various engineering fields [54], [55]. The HGD comprises two main parts i.e. the wave generation subsystem and the gear transmission subsystem and can be further decomposed into 5 components. This HGD is still in a prototype phase and its reliability characteristics have not been sufficiently discovered yet. Our aim is to use dependent failure time data to assess its reliability taking the model uncertainty into account. In particular, the working mechanism of the wave generation subsystem is well-studied and hence its configuration is fully specified in this BN model. Comparatively, the gear transmission subsystem is newly developed so that its reliability dependence on other parts of the system needs to be investigated. For mathematical convenience, we use the BN shown in Fig. 15 to model the HGD device (more details regarding the HGD device refer [54], [55]). Note that this BN contains a hybrid structure as an intra-level connection (from $S_{1} \rightarrow S_{2}$ ), which means that most existing methods cannot be applied. 


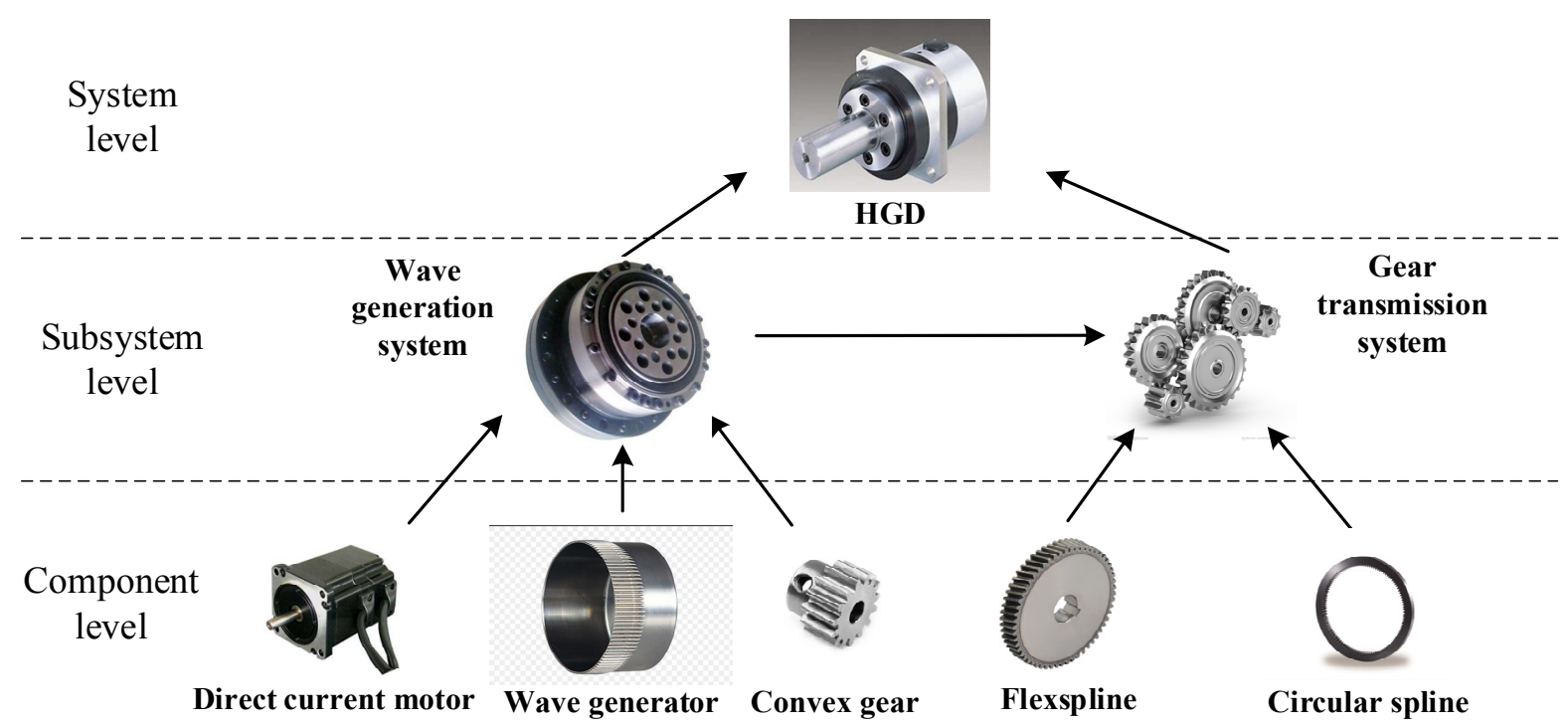

Fig. 14 Harmonic Gear Drive (HGD) device

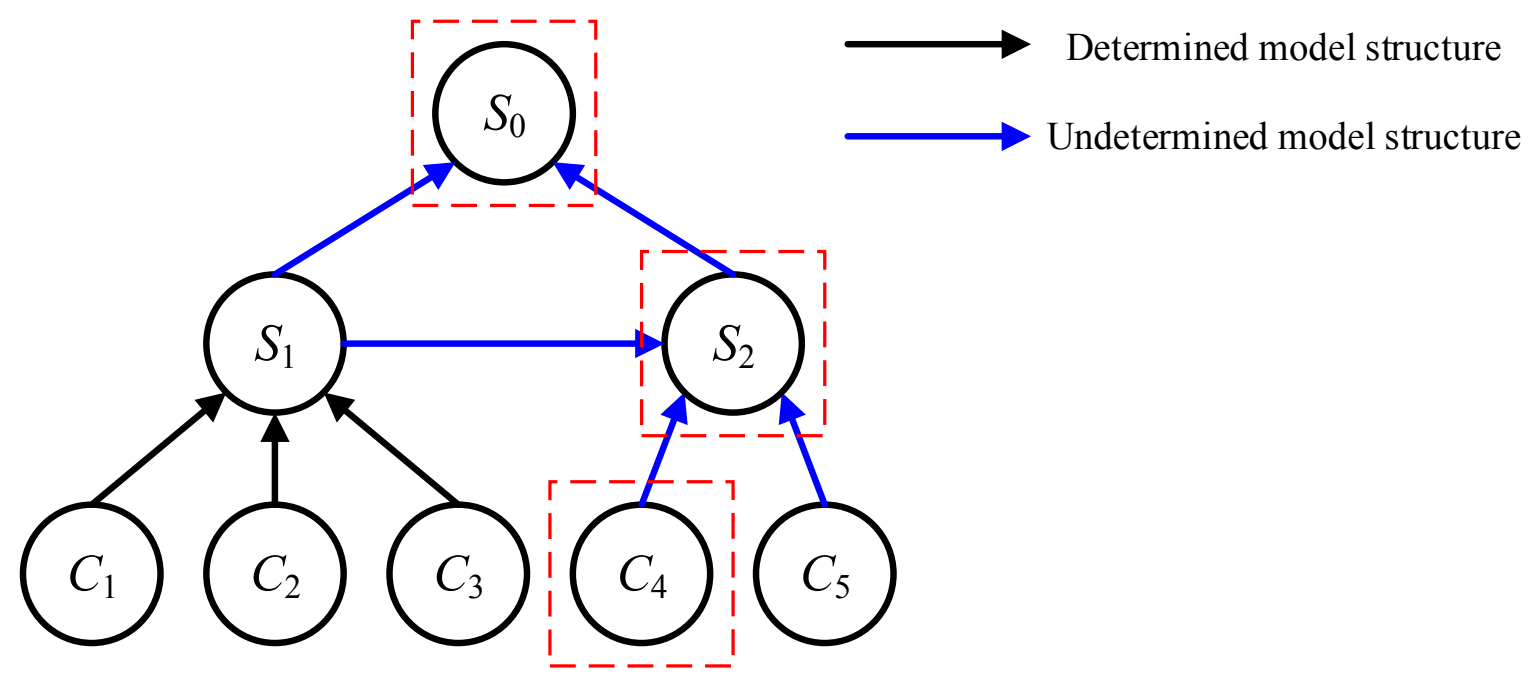

Fig. 15 BN model representing the HGD device

For detailed parameter specifications, the lifetime distributions of three components $C_{1}, C_{2}, C_{4}$ are modeled as Exponential distributions with parameters $\lambda_{1} \lambda_{2} \lambda_{4}$ respectively. Weibull models are adopted for $C_{3}, C_{5}$. To imitate a non-informative scenario, we assign uniform prior distributions to all unknown lifetime model parameters in Table 1 and CPT parameters in Table 2. In total, 10 independent reliability tests are conducted to collect the failure time data. For each individual test, three sensors are installed to monitor the working states and record the failure time and hence produce three dependent data points. The mixed dataset representing all available data is summarized in Table 3. 
Table 1 Lifetime model parameter settings

\begin{tabular}{|c|c|c|c|}
\hline Component & Life time model & Parameter & Prior distribution \\
\hline$C_{1}$ & Exponential & $\lambda_{1}$ & Uniform $(0,0.03)$ \\
\hline$C_{2}$ & Exponential & $\lambda_{2}$ & Uniform $(0.05,0.15)$ \\
\hline \multirow{2}{*}{$C_{3}$} & \multirow{2}{*}{ Weibull } & $\beta_{3}$ & Uniform $(2.5,3)$ \\
\hline & & $\eta_{3}$ & Uniform $(110,140)$ \\
\hline$C_{4}$ & Exponential & $\lambda_{2}$ & Uniform(0,0.007) \\
\hline \multirow{2}{*}{$C_{5}$} & \multirow{2}{*}{ Weibull } & $\beta_{5}$ & Uniform $(2,2.5)$ \\
\hline & & $\eta_{5}$ & Uniform $(40,60)$ \\
\hline
\end{tabular}

$\operatorname{Pr}\left(S_{0}=0 \mid S_{1}=0, S_{2}=0\right)=p_{00} \operatorname{Pr}\left(S_{2}=0 \mid S_{1}=0, C_{4}=0, C_{5}=0\right)=p_{000} \operatorname{Pr}\left(S_{2}=0 \mid S_{1}=1, C_{4}=1, C_{5}=0\right)=p_{110}$ $\operatorname{Pr}\left(S_{0}=0 \mid S_{1}=1, S_{2}=0\right)=p_{10} \operatorname{Pr}\left(S_{2}=0 \mid S_{1}=1, C_{4}=0, C_{5}=0\right)=p_{100} \quad \operatorname{Pr}\left(S_{2}=0 \mid S_{1}=1, C_{4}=0, C_{5}=1\right)=p_{101}$ $\operatorname{Pr}\left(S_{0}=0 \mid S_{1}=0, S_{2}=1\right)=p_{01} \operatorname{Pr}\left(S_{2}=0 \mid S_{1}=0, C_{4}=1, C_{5}=0\right)=p_{010} \quad \operatorname{Pr}\left(S_{2}=0 \mid S_{1}=0, C_{4}=1, C_{5}=1\right)=p_{011}$ $\operatorname{Pr}\left(S_{0}=0 \mid S_{1}=1, S_{2}=1\right)=p_{11} \quad \operatorname{Pr}\left(S_{2}=0 \mid S_{1}=0, C_{4}=0, C_{5}=1\right)=p_{001} \quad \operatorname{Pr}\left(S_{2}=0 \mid S_{1}=1, C_{4}=1, C_{5}=1\right)=p_{111}$

Table 2 Prior distributions for CPT parameters

\begin{tabular}{|c|c|c|c|c|c|}
\hline CPT & Prior & CPT & Prior & CPT & Prior \\
Parameter & distribution & Parameter & distribution & Parameter & distribution \\
\hline$p_{00}$ & Unif $(0.75,1)$ & $p_{000}$ & Unif $(0.75,1)$ & $p_{110}$ & Unif $(0.25,0.5)$ \\
\hline$p_{10}$ & Unif $(0.25,0.5)$ & $p_{100}$ & Unif $(0.5,0.75)$ & $p_{101}$ & Unif $(0.25,0.5)$ \\
\hline$p_{01}$ & Unif $(0.25,0.5)$ & $p_{010}$ & Unif $(0.5,0.75)$ & $p_{011}$ & Unif $(0.25,0.5)$ \\
\hline$p_{11}$ & Unif $(0,0.25)$ & $p_{001}$ & Unif $(0.5,0.57)$ & $p_{111}$ & Unif $(0,0.25)$ \\
\hline
\end{tabular}

Table 3 Failure time records

\begin{tabular}{|c|c|c|c|c|}
\hline & \multirow{2}{*}{ Test \# } & \multicolumn{3}{|c|}{ Dependent data } \\
\hline & & $\mathbf{t}_{S_{0}}$ & $\mathbf{t}_{S_{2}}$ & $\mathbf{t}_{C_{4}}$ \\
\hline \multirow{7}{*}{ 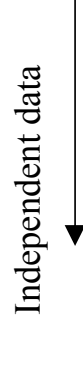 } & 1 & 61 & 60 & 58 \\
\hline & 2 & 42 & 41 & 41 \\
\hline & 3 & 63 & 63 & 63 \\
\hline & 4 & 55 & 53 & 50 \\
\hline & 5 & 76 & 76 & 72 \\
\hline & 6 & 67 & 67 & 66 \\
\hline & 7 & 83 & 75 & 75 \\
\hline
\end{tabular}




\begin{tabular}{|c|c|c|c|c|}
\hline \multirow{3}{*}{} & 8 & 88 & 77 & 74 \\
\cline { 2 - 5 } & 9 & 57 & 50 & 49 \\
\cline { 2 - 5 } & 10 & 60 & 59 & 59 \\
\hline
\end{tabular}

6.2 Parameter estimation and reliability analysis

By introducing the notions of EEL and IEL function, the likelihood contributions of each individual evidence can be identified. The EEL function of component 4 is given as

$$
L\left(t_{C_{4}}\right)=f_{C_{4}}(t)=\lambda_{4} e^{-\lambda_{4} t_{C_{4}}}
$$

As the IEL function is dependent on both explicit evidence and implicit evidence, the expressions of two IEL

functions are consequently case-based. We give the general form of the two IEL functions as Eq. (52) and (53).

$$
\begin{aligned}
& L\left(t_{S_{2}} \mid t_{C_{4}}\right)=\left\{\begin{array}{cl}
\frac{\partial F_{S_{2}}(t)}{\partial F_{C_{4}}(t)} & \text { if } t_{S_{2}}=t_{C_{4}} \\
\sum_{j=1,2,3,5} \frac{\partial F_{S_{2}}(t)}{\partial F_{C_{j}}(t)} \times f_{C_{j}}(t) & \text { otherwise }
\end{array}\right. \\
& \int\left(\begin{array}{l}
\left(p_{000}-p_{010}\right) F_{S_{1}} F_{C_{5}}+\left(p_{100}-p_{110}\right)\left(1-F_{S_{1}}\right) F_{C_{5}} \\
+\left(p_{001}-p_{011}\right) F_{S_{1}}\left(1-F_{C_{5}}\right)+\left(p_{101}-p_{111}\right)\left(1-F_{S_{1}}\right)\left(1-F_{C_{5}}\right)
\end{array}\right) l_{t=t_{S_{2}}} \text { if } t_{S_{2}}=t_{C_{4}}
\end{aligned}
$$

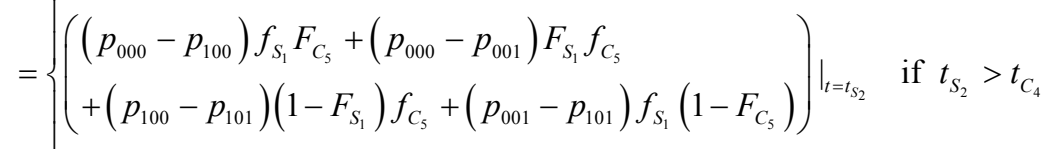

$$
\begin{aligned}
& \left.\left(\begin{array}{l}
\left(p_{010}-p_{110}\right) f_{S_{1}} F_{C_{5}}+\left(p_{010}-p_{011}\right) F_{S_{1}} f_{C_{5}} \\
+\left(p_{110}-p_{111}\right) p_{110}\left(1-F_{S_{1}}\right) f_{C_{5}}+\left(p_{011}-p_{111}\right) f_{S_{1}}\left(1-F_{C_{5}}\right)
\end{array}\right)\right|_{t=t_{S_{2}}} \quad \text { if } t_{S_{2}}<t_{C_{4}} \\
& L\left(t_{S_{0}} \mid t_{S_{2}}\right)=\left\{\begin{array}{cc}
\frac{\partial F_{S_{0}}(t)}{\partial F_{S_{2}}(t)} & \text { if } t_{S_{0}}=t_{S_{2}} \\
\sum_{j=1,2,3} \frac{\partial F_{S_{0}}(t)}{\partial F_{C_{j}}(t)} \times f_{C_{j}}(t) & \text { otherwise }
\end{array}\right. \\
& = \begin{cases}{\left.\left[\left(p_{00}-p_{01}\right) F_{S_{1}}+\left(p_{10}-p_{11}\right)\left(1-F_{S_{1}}\right)\right]\right|_{t=t_{S_{0}}}} & \text { if } t_{S_{0}}=t_{S_{2}} \\
\left.\left(p_{00}-p_{10}\right) f_{S_{1}}\right|_{t=t_{S_{0}}} & \text { if } t_{S_{0}}>t_{S_{2}} \\
\left.\left(p_{01}-p_{11}\right) f_{S_{1}}\right|_{t=t_{S_{0}}} & \text { if } t_{S_{0}}<t_{S_{2}}\end{cases}
\end{aligned}
$$

where $F_{S_{1}}(t)=F_{C_{1}}(t) F_{C_{2}}(t) F_{C_{3}}(t)$. The overall likelihood is calculated from a multiplication of all likelihood contributions from both independent and dependent evidence as Eq. (54)

$$
L\left(\mathbf{t}_{C_{4}}, \mathbf{t}_{S_{2}}, \mathbf{t}_{S_{0}}\right)=\prod_{i=1}^{10} L\left(t_{C_{4}}^{i}\right) L\left(t_{S_{2}}^{i} \mid t_{C_{4}}^{i}\right) L\left(t_{S_{0}}^{i} \mid t_{S_{2}}^{i}\right)
$$


With the given prior distributions, the posterior distribution of unknown parameters are estimated using the MCMC algorithm in the software OpenBUGS.

$$
\pi(\boldsymbol{\theta}, \mathbf{p} \mid E) \propto L(E \mid \boldsymbol{\theta}, \mathbf{p}) \pi_{0}(\boldsymbol{\theta}, \mathbf{p})
$$

where the distribution parameters vector $\boldsymbol{\theta}=\left(\lambda_{1} \lambda_{2}, \beta_{3}, \eta_{3}, \lambda_{4}, \beta_{5}, \eta_{5}\right)$ and the CPT parameters vector $\mathbf{p}=\left(p_{00}, p_{10}, p_{01}\right.$, $\left.p_{11}, p_{000}, p_{001}, p_{010}, p_{100}, p_{110}, p_{101}, p_{011}, p_{111}\right)$

6.3 Results and discussion

A total of $10^{5}$ samples are generated from the joint posterior distribution with the first half $5 \times 10^{4}$ samples for burn-in. The remaining samples are thinned by selecting every 5th observation to reduce the autocorrelation between adjacent samples. To ensure the generated posterior samples converge to the target distribution, we employ the Raftery-Lewis (RL) diagnostic to determine when the MCMC samples converge to stationary posterior distributions. It is found all unknown parameters approach to their stationary state after $5 \times 10^{4}$ iterations. The estimation results are presented in Table 4 and Table 5.

Table 4 Statistics of the posterior samples for distribution parameters

\begin{tabular}{|c|c|c|c|c|c|c|c|}
\hline Parameter & Mean & SD & $2.5 \%$ & $25 \%$ & Median & $75 \%$ & $97.5 \%$ \\
\hline$\lambda_{1}$ & 0.0243 & 0.004348 & 0.01415 & 0.02164 & 0.02524 & 0.02784 & 0.02979 \\
\hline$\lambda_{2}$ & 0.09985 & 0.0285 & 0.05244 & 0.07563 & 0.09998 & 0.1242 & 0.1475 \\
\hline$\beta_{3}$ & 2.716 & 0.1387 & 2.51 & 2.597 & 2.702 & 2.827 & 2.98 \\
\hline$\eta_{3}$ & 115.7 & 5.392 & 110.1 & 111.6 & 114.0 & 118.1 & 130.0 \\
\hline$\lambda_{4}$ & 0.006194 & $6.951 \mathrm{E}-4$ & 0.00442 & 0.005841 & 0.006382 & 0.006733 & 0.006976 \\
\hline$\beta_{5}$ & 2.275 & 0.1429 & 2.017 & 2.155 & 2.291 & 2.399 & 2.49 \\
\hline$\eta_{5}$ & 53.28 & 4.787 & 42.62 & 50.04 & 54.09 & 57.34 & 59.73 \\
\hline
\end{tabular}

Table 5 Statistics of the posterior samples for CPT parameters

\begin{tabular}{|c|c|c|c|c|c|c|c|}
\hline Parameter & Mean & SD & $2.5 \%$ & $25 \%$ & Median & $75 \%$ & $97.5 \%$ \\
\hline$p_{00}$ & 0.9383 & 0.05445 & 0.7949 & 0.9109 & 0.9544 & 0.9808 & 0.9983 \\
\hline
\end{tabular}




\begin{tabular}{|c|c|c|c|c|c|c|c|}
\hline$p_{10}$ & 0.3796 & 0.09546 & 0.2559 & 0.304 & 0.3599 & 0.4355 & 0.6123 \\
\hline$p_{01}$ & 0.4637 & 0.1399 & 0.2585 & 0.3414 & 0.4488 & 0.5735 & 0.7302 \\
\hline$p_{11}$ & 0.08539 & 0.0642 & 0.002799 & 0.0312 & 0.072 & 0.1281 & 0.2302 \\
\hline$p_{000}$ & 0.9215 & 0.06185 & 0.7774 & 0.8815 & 0.9368 & 0.9725 & 0.9975 \\
\hline$p_{100}$ & 0.6818 & 0.05547 & 0.5396 & 0.6509 & 0.6962 & 0.7254 & 0.7476 \\
\hline$p_{010}$ & 0.6189 & 0.07158 & 0.5064 & 0.556 & 0.6154 & 0.6798 & 0.7432 \\
\hline$p_{001}$ & 0.6321 & 0.07221 & 0.5067 & 0.57 & 0.6366 & 0.695 & 0.7446 \\
\hline$p_{110}$ & 0.3344 & 0.06422 & 0.2527 & 0.2802 & 0.3197 & 0.3787 & 0.4775 \\
\hline$p_{101}$ & 0.3327 & 0.0619 & 0.2527 & 0.2805 & 0.3201 & 0.3746 & 0.4721 \\
\hline$p_{011}$ & 0.3744 & 0.0714 & 0.2573 & 0.3133 & 0.3739 & 0.436 & 0.4936 \\
\hline$p_{111}$ & 0.1058 & 0.07049 & 0.004039 & 0.04449 & 0.09629 & 0.1633 & 0.2393 \\
\hline
\end{tabular}

The evaluated distribution parameters in Table 4 can be further used to predict the reliability for a node of interest, meanwhile, the evaluated CPT parameters in Table 5 can help us assess how composing components affecting the working state of higher-level nodes. For example, it is observed that the mean of $p_{01}=0.3796<p_{10}=$ 0.4637 suggesting subsystem 1 plays a more important role than subsystem 2 for the functioning of the whole system. Besides the discovery of the reliability relationship between inter-level nodes, the proposed method is capable of capturing the intra-level dependence. It is found the mean of $p_{011}=0.3744>p_{110}=0.3344$ and the mean of $p_{011}=$ $0.3744>p_{101}=0.3327$, which indicates the subsystem $S_{1}$ has more effect on the working state of subsystem $S_{2}$ compared with the two components $C_{4}$ and $C_{5}$. Comparably, existing studies can barely catch this dependence as their methodologies are not applied to a BN with hybrid structures. The proposed method can efficiently extract the dependence information embedded within the simultaneously collected data set and produce valuable estimation results. By exploiting this information, the dependencies among multi-level nodes are progressively revealed.

Another interesting thing is that the CPT parameter $p_{00}$ has a non-zero value which indicates a failure chance for $S_{0}$ when both $S_{1}$ and $S_{2}$ are functioning well. These findings are also applied to $p_{000}$ indicating that $S_{2}$ has a failure chance when all its direct predecessor nodes $S_{1}, C_{4}, C_{5}$ are functioning well. This is impossible to occur in a 
deterministic reliability model such as a RBD or a FT. However, for a BN model, its variability allows the inclusion of such uncertainty. This means that, there must be some undiscovered physical factors affecting the reliability of $S_{0}$ and $S_{2}$ besides the presented nodes and further investigation should be taken into consideration to reduce such uncertainty.

For the system reliability analysis, Eq. (20) is adopted to calculate the reliability for a node of interest for a given time-point. We calculate the reliability of all nodes for every 10 time units and present the reliability curves changing over time in Fig. 16. A radar map is employed to give quantitative comparisons of reliability for all nodes at three time-points (i.e. $t=0,10,100$ ) as shown in Fig. 17.

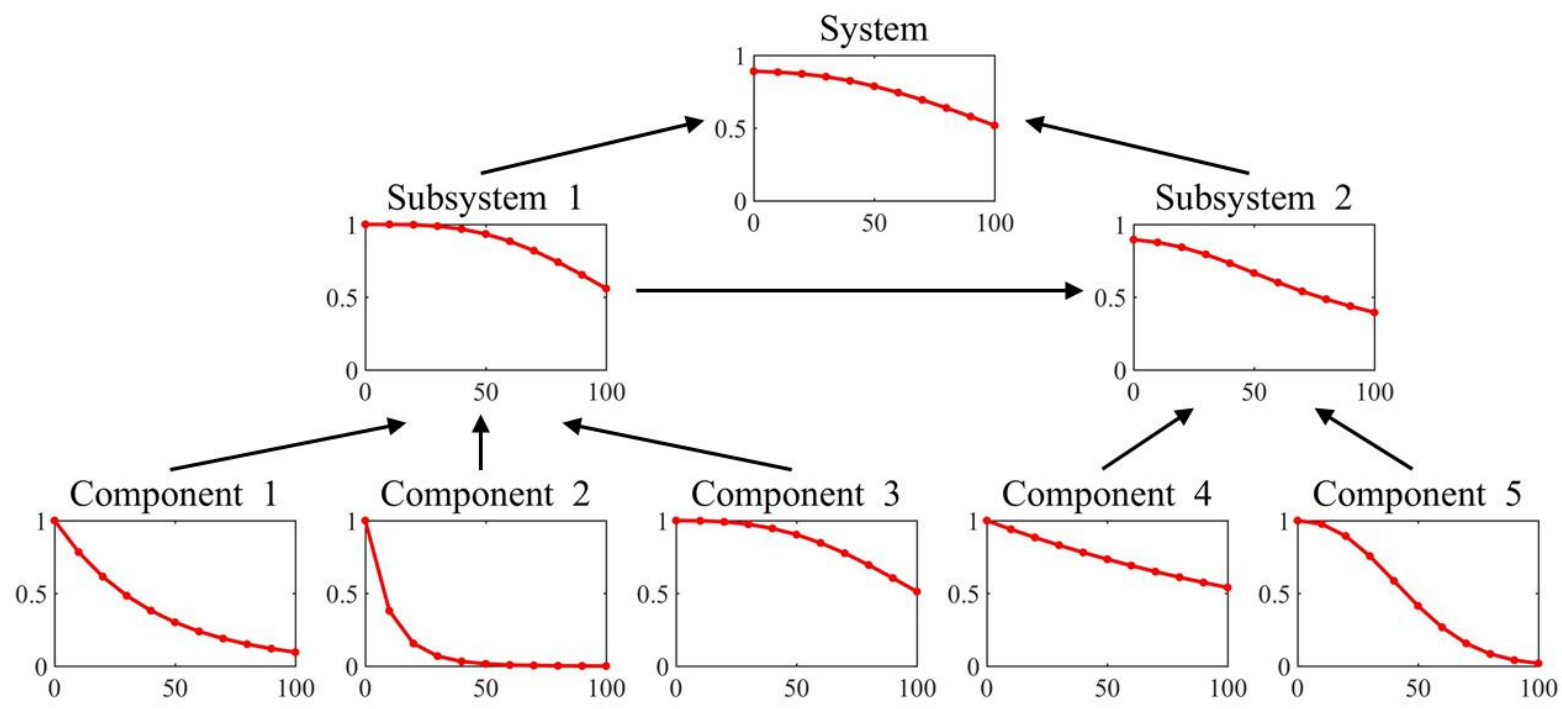

Fig. 16 Reliability predictions for the HGD
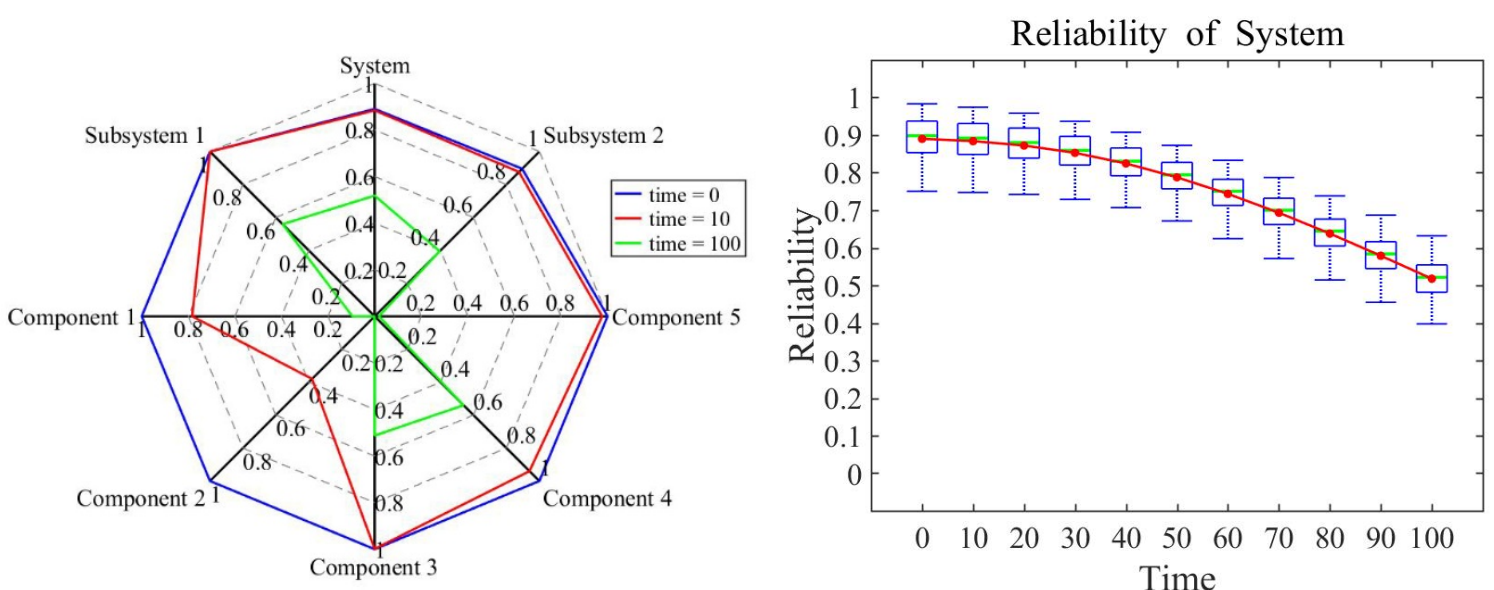

Fig. 17 Radar map of the predicted reliability for all nodes 

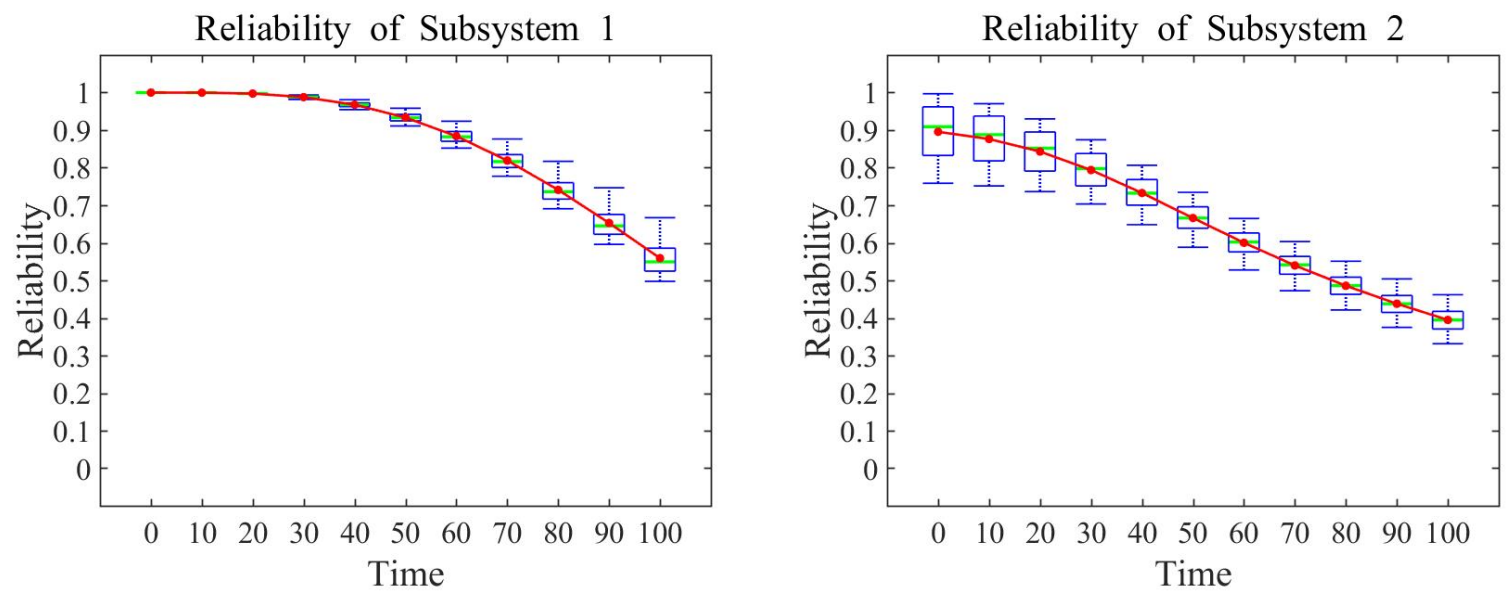

Fig. 18 Reliability changes over time for $S_{1}$ (left) and $S_{2}$ (right)
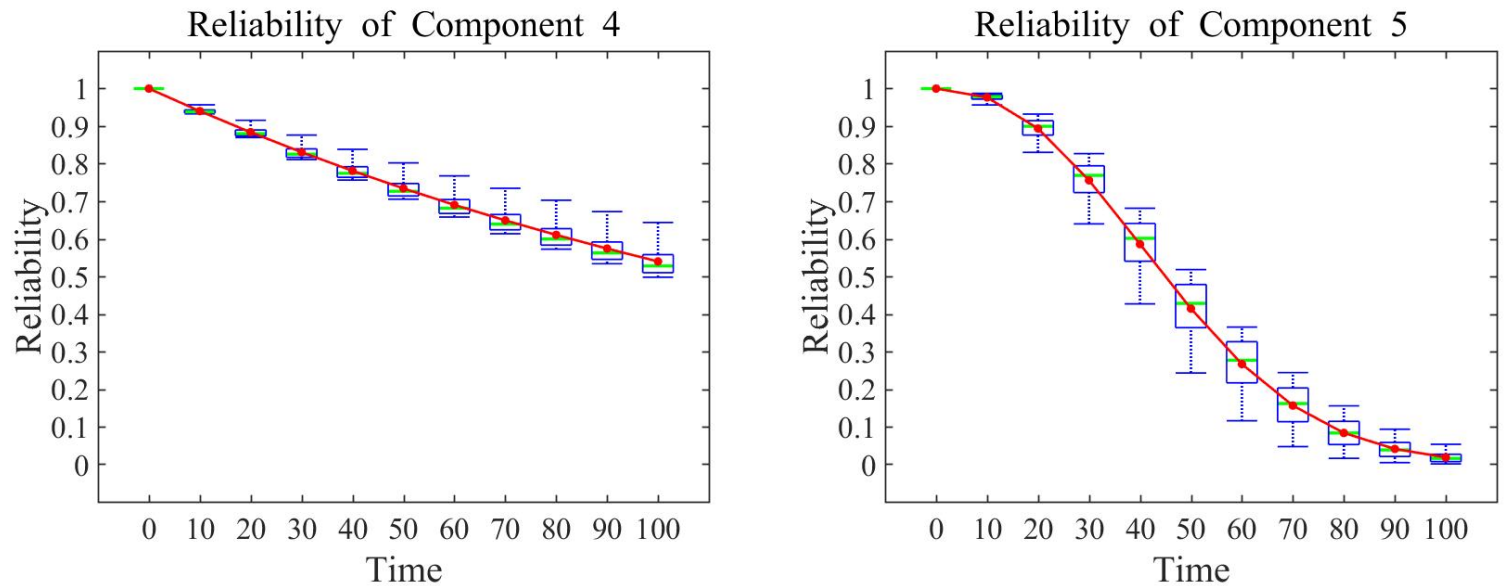

Fig. 19 Reliability changes over time for $C_{4}$ (left) and $C_{5}$ (right)

Another benefit of our approach is that it can be used to estimate the parameters of both observed and unobserved nodes. We show the updated reliability curves of the $S_{1}$ (unobserved node), $S_{2}$ (observed node) in Fig. 18, and $C_{4}$ (observed node), $C_{5}$ (unobserved node) in Fig. 19 respectively. This has profound meanings in practical engineering since the installation of sensors to many crucial components is sometimes intractable. Benefiting from a successful multi-source information extraction \& aggregation, we can compensate for the inadequate information of a vital component and update our prior belief on this component even without explicit evidence. The proposed method is essentially analytic for system reliability analysis and it requires many assumptions for engineering use. For demonstration purpose, we adopt Exponential \& Weibull distributions to model the failure time data in this application example. However, these assumptions are quite strong and should be carefully reviewed in other engineering cases. Also, numerical simulation errors and convergence performance of the generated Markov Chain 
should not be overlooked.

\section{Conclusions and future works}

This paper proposed a full Bayesian approach for system reliability evaluation in the presence of dependent data. A likelihood decomposition method is developed to separate the overall joint likelihood into several conditionally independent likelihoods. The concepts of Explicit Evidence-based Likelihood and Implicit Evidencebased Likelihood are established to distinguish different contributions of outer source information in the likelihood construction. An inferential diagram is further developed as an intuitive tool to generate the implicit evidence. The application field of our approach is not only limited to deterministic system models such as fault tree models or event trees but also includes hierarchical BNs and a BN with hybrid structure, which promises a brighter engineering application prospect.

Several numerical cases and a practical engineering case are demonstrated to validate the proposed method and to show its superiority. The estimation results show that our approach is capable of capturing the dependence information embedded within the simultaneously collected test data and then can progressively reveal the dependencies between inter-level nodes and intra-level nodes. Another benefit of our approach is that it could be used to estimate the parameters of unobserved nodes. This is meaningful in practical engineering since collecting data from all crucial components is not always accessible. This approach helps us in providing a better understanding of the nature of dependent evidence in system reliability analysis.

The proposed method is developed for a static Bayesian model with continuous lifetime data. Extending it to a dynamic $\mathrm{BN}$ with heterogeneous data is in the consideration for our future works. The challenges mainly comes from the construction of inferential diagrams as the procedure is time-consuming, even intractable, given timedependent structure and/or evidences. Moreover, as the accumulation of multi-source information continuously 
grows in practical engineering cases, the presence of imprecise conflicting information is not rare. In this situation, establishing a coherent reliability approach taking the imprecise and/or inconsistent information into account can significantly benefit the reliability assessment of complex systems and hence produce trustworthy results with less uncertainty. To meet this demand, developing a comprehensive Bayesian approach for complex systems with both imprecise and inconsistent information could be another research interest for our future works.

\section{Acknowledgment}

This work is partially supported by the National Natural Science Foundation of China under Grant 52005032, 72071005, the Aeronautical Science Foundation of China under Grant 2018ZC74001, the Fundamental Research Funds for the Central Universities of China under Grant FRF-TP-20-008A2 and the China Scholarship Council (CSC) under Grant 201906465064.

\section{Reference}

[1] G. $\mathrm{Wu}, \mathrm{Z}$. Li, and P. Liu, "Risk-informed reliability improvement optimization for verification and validation planning based on set covering modeling," Proc. Inst. Mech. Eng. Part O J. Risk Reliab., 2020, doi: 10.1177/1748006X19898294.

[2] M. Beer, S. Ferson, and V. Kreinovich, "Imprecise probabilities in engineering analyses," Mech. Syst. Signal Process., vol. 37, no. 1-2, pp. 4-29, 2013, doi: 10.1016/j.ymssp.2013.01.024.

[3] M. Beer, Y. Zhang, S. T. Quek, and K. K. Phoon, "Reliability analysis with scarce information: Comparing alternative approaches in a geotechnical engineering context," Struct. Saf., vol. 41, pp. 1-10, 2013, doi: 10.1016/j.strusafe.2012.10.003.

[4] D. Straub, R. Schneider, E. Bismut, and H. J. Kim, "Reliability analysis of deteriorating structural systems," Struct. Saf., vol. 82, no. September 2019, p. 101877, 2020, doi: 10.1016/j.strusafe.2019.101877.

[5] L. Yang, Y. Guo, and Q. Wang, "Reliability Assessment of a Hierarchical System Subjected to Inconsistent Priors and Multilevel Data," IEEE Trans. Reliab., vol. 69, no. 1, pp. 277-292, Mar. 2020, doi: 10.1109/TR.2019.2895501.

[6] D. Straub and I. Papaioannou, "Bayesian Updating with Structural Reliability Methods," J. Eng. Mech., vol. 141, no. 3, p. 04014134, 2015, doi: 10.1061/(asce)em.1943-7889.0000839.

[7] D. Straub, I. Papaioannou, and W. Betz, "Bayesian analysis of rare events," J. Comput. Phys., vol. 314, pp. 538-556, 2016, doi: 10.1016/j.jcp.2016.03.018.

[8] Z. Li, Y. Deng, and C. Mastrangelo, "Model selection for degradation-based Bayesian reliability analysis," J. Manuf. Syst., vol. 37, pp. 72-82, 2015, doi: 10.1016/j.jmsy.2015.09.005.

[9] D. R. Insua, F. Ruggeri, R. Soyer, and S. Wilson, "Advances in Bayesian decision making in reliability," 
Eur. J. Oper. Res., vol. 282, no. 1, pp. 1-18, 2020, doi: 10.1016/j.ejor.2019.03.018.

[10] A. Yazdani, M. S. Shahidzadeh, and T. Takada, "Bayesian networks for disaggregation of structural reliability,” Struct. Saf., vol. 82, no. April 2019, p. 101892, 2020, doi: 10.1016/j.strusafe.2019.101892.

[11] D. Lee and R. Pan, "A nonparametric Bayesian network approach to assessing system reliability at early design stages," Reliab. Eng. Syst. Saf., vol. 171, no. November 2017, pp. 57-66, 2018, doi: 10.1016/j.ress.2017.11.009.

[12] H. F. Martz and R. A. Waller, "Bayesian reliability analysis of complex series/parallel systems of binomial subsystems and components," Technometrics, vol. 32, no. 4, pp. 407-416, 1990, doi: 10.1080/00401706.1990.10484727.

[13] V. E. Johnson, A. Moosman, and P. Cotter, “A hierarchical model for estimating the early reliability of complex systems," IEEE Trans. Reliab., vol. 54, no. 2, pp. 224-231, 2005, doi: 10.1109/TR.2005.847262.

[14] M. Hamada, H. F. Martz, C. S. Reese, T. Graves, V. Johnson, and A. G. Wilson, “A fully Bayesian approach for combining multilevel failure information in fault tree quantification and optimal follow-on resource allocation," Reliab. Eng. Syst. Saf., vol. 86, no. 3, pp. 297-305, 2004, doi: 10.1016/j.ress.2004.02.001.

[15] T. L. Graves, M. S. Hamada, R. Klamann, A. Koehler, and H. F. Martz, “A fully Bayesian approach for combining multi-level information in multi-state fault tree quantification," Reliab. Eng. Syst. Saf., vol. 92, no. 10, pp. 1476-1483, 2007, doi: 10.1016/j.ress.2006.11.001.

[16] D. Straub and A. Der Kiureghian, "Bayesian Network Enhanced with Structural Reliability Methods: Methodology," J. Eng. Mech., vol. 136, no. 10, pp. 1248-1258, 2010, doi: 10.1061/(asce)em.19437889.0000173 .

[17] D. Straub and A. Der Kiureghian, "Bayesian Network Enhanced with Structural Reliability Methods: Application," J. Eng. Mech., vol. 136, no. 10, pp. 1259-1270, 2010, doi: 10.1061/(asce)em.19437889.0000170 .

[18] J. Guo, Z. Steven Li, and J. Judy Jin, "System reliability assessment with multilevel information using the Bayesian melding method," Reliab. Eng. Syst. Saf., vol. 170, no. November 2016, pp. 146-158, 2018, doi: 10.1016/j.ress.2017.09.020.

[19] J. GUO, Z. LI, and T. KEYSER, “A Bayesian approach for integrating multilevel priors and data for aerospace system reliability assessment," Chinese J. Aeronaut., vol. 31, no. 1, pp. 41-53, 2018, doi: 10.1016/j.cja.2017.08.020.

[20] J. Guo, H. Z. Huang, W. Peng, and J. Zhou, "Bayesian information fusion for degradation analysis of deteriorating products with individual heterogeneity," Proc. Inst. Mech. Eng. Part O J. Risk Reliab., vol. 233, no. 4, pp. 615-622, 2019, doi: 10.1177/1748006X18808964.

[21] C. Shane Reese, A. G. Wilson, J. Guo, M. S. Hamada, and V. E. Johnson, "A Bayesian model for integrating multiple sources of lifetime information in system-reliability assessments," J. Qual. Technol., vol. 43, no. 2, pp. 127-141, 2011, doi: 10.1080/00224065.2011.11917851.

[22] A. G. Wilson, C. M. Anderson-Cook, and A. V. Huzurbazar, "A case study for quantifying system reliability and uncertainty," Reliab. Eng. Syst. Saf., vol. 96, no. 9, pp. 1076-1084, 2011, doi: 10.1016/j.ress.2010.09.012.

[23] J. Guo and A. G. Wilson, "Bayesian methods for estimating system reliability using heterogeneous multilevel information," Technometrics, vol. 55, no. 4, pp. 461-472, 2013, doi: 10.1080/00401706.2013.804441.

[24] M. Li, H. Meng, and Q. Zhang, "A nonparametric Bayesian modeling approach for heterogeneous lifetime data with covariates," Reliab. Eng. Syst. Saf., vol. 167, no. May, pp. 95-104, 2017, doi: 
10.1016/j.ress.2017.05.029.

[25] M. Li, J. Han, and J. Liu, "Bayesian nonparametric modeling of heterogeneous time-to-event data with an unknown number of sub-populations," IISE Trans., vol. 49, no. 5, pp. 481-492, 2017, doi: 10.1080/0740817X.2016.1234732.

[26] M. Li, W. Zhang, Q. Hu, H. Guo, and J. Liu, "Design and risk evaluation of reliability demonstration test for hierarchical systems with multilevel information aggregation," IEEE Trans. Reliab., vol. 66, no. 1, pp. 135-147, 2017, doi: 10.1109/TR.2016.2619689.

[27] A. Namdari and Z. (Steven) Li, “A review of entropy measures for uncertainty quantification of stochastic processes," Adv. Mech. Eng., vol. 11, no. 6, pp. 1-14, 2019, doi: 10.1177/1687814019857350.

[28] J. Guo, Z. Li, and M. Li, “A Review on Prognostics Methods for Engineering Systems," IEEE Trans. Reliab., vol. 69, no. 3, pp. 1110-1129, 2020, doi: 10.1109/TR.2019.2957965.

[29] F. P. A. Coolen, T. Coolen-Maturi, and A. H. Al-nefaiee, "Nonparametric predictive inference for system reliability using the survival signature," Proc. Inst. Mech. Eng. Part O J. Risk Reliab., vol. 228, no. 5, pp. 437-448, 2014, doi: 10.1177/1748006X14526390.

[30] G. Feng, E. Patelli, M. Beer, and F. P. A. Coolen, "Imprecise system reliability and component importance based on survival signature," Reliab. Eng. Syst. Saf., vol. 150, pp. 116-125, 2016, doi: 10.1016/j.ress.2016.01.019.

[31] E. Patelli, G. Feng, F. P. A. Coolen, and T. Coolen-Maturi, "Simulation methods for system reliability using the survival signature," Reliab. Eng. Syst. Saf., vol. 167, no. June, pp. 327-337, 2017, doi: 10.1016/j.ress.2017.06.018.

[32] L. J. M. Aslett, F. P. A. Coolen, and S. P. Wilson, "Bayesian Inference for Reliability of Systems and Networks Using the Survival Signature," Risk Anal., vol. 35, no. 9, pp. 1640-1651, 2015, doi: $10.1111 /$ risa. 12228 .

[33] G. Walter, L. J. M. Aslett, and F. P. A. Coolen, "Bayesian nonparametric system reliability using sets of priors,” Int. J. Approx. Reason., vol. 80, pp. 67-88, 2017, doi: 10.1016/j.ijar.2016.08.005.

[34] G. Walter and F. P. A. Coolen, "Robust Bayesian Reliability for Complex Systems under Prior-Data Conflict," ASCE-ASME J. Risk Uncertain. Eng. Syst. Part A Civ. Eng., vol. 4, no. 3, p. 04018025, 2018, doi: 10.1061/ajrua6.0000974.

[35] T. L. Graves, M. S. Hamada, R. M. Klamann, A. C. Koehler, and H. F. Martz, "Using simultaneous higherlevel and partial lower-level data in reliability assessments," Reliab. Eng. Syst. Saf., vol. 93, no. 8, pp. 1273-1279, 2008, doi: 10.1016/j.ress.2007.07.002.

[36] K. O. Kim, "Bayesian reliability when system and subsystem failure data are obtained in the same time period,” J. Korean Stat. Soc., vol. 42, no. 1, pp. 95-103, 2013, doi: 10.1016/j.jkss.2012.05.004.

[37] C. Jackson and A. Mosleh, "Downwards inference: Bayesian analysis of overlapping higher-level data sets of complex binary-state on-demand systems," Proc. Inst. Mech. Eng. Part O J. Risk Reliab., vol. 226, no. 2, pp. 182-193, 2012, doi: 10.1177/1748006X11402423.

[38] C. Jacksonn and A. Mosleh, "Bayesian inference with overlapping data: Methodology for reliability estimation of multi-state on-demand systems," Proc. Inst. Mech. Eng. Part O J. Risk Reliab., vol. 226, no. 3, pp. 283-294, 2012, doi: 10.1177/1748006X11424104.

[39] C. Jackson and A. Mosleh, "Bayesian inference with overlapping data for systems with continuous life metrics,” Reliab. Eng. Syst. Saf., vol. 106, pp. 217-231, 2012, doi: 10.1016/j.ress.2012.04.006.

[40] C. Jackson and A. Mosleh, "Bayesian inference with overlapping data: Reliability estimation of multistate on-demand continuous life metric systems with uncertain evidence," Reliab. Eng. Syst. Saf., vol. 145, pp. 124-135, 2016, doi: 10.1016/j.ress.2015.09.006. 
[41] T. L. Graves and M. S. Hamada, "A Note on Incorporating Simultaneous Multi-level Failure Time Data in System Reliability Assessments," Qual. Reliab. Eng. Int., vol. 32, no. 3, pp. 1127-1135, 2016, doi: 10.1002/qre.1820.

[42] S. W. Lin, Y. T. Liu, and M. A. Jerusalem, "Bayesian reliability analysis of a products of probabilities model for parallel systems with dependent components," Int. J. Prod. Res., vol. 56, no. 4, pp. 1521-1532, 2018, doi: $10.1080 / 00207543.2017 .1358468$.

[43] C. Guo, F. Khan, and S. Imtiaz, "Risk assessment of process system considering dependencies," J. Loss Prev. Process Ind., vol. 55, no. April, pp. 204-212, 2018, doi: 10.1016/j.jlp.2018.06.014.

[44] N. Khakzad, F. Khan, and P. Amyotte, "Quantitative risk analysis of offshore drilling operations: A Bayesian approach,”Saf. Sci., vol. 57, pp. 108-117, 2013, doi: 10.1016/j.ssci.2013.01.022.

[45] M. Abimbola, F. Khan, N. Khakzad, and S. Butt, "Safety and risk analysis of managed pressure drilling operation using Bayesian network,” Saf. Sci., vol. 76, pp. 133-144, 2015, doi: 10.1016/j.ssci.2015.01.010.

[46] N. Khakzad, "Application of dynamic Bayesian network to risk analysis of domino effects in chemical infrastructures," Reliab. Eng. Syst. Saf., vol. 138, pp. 263-272, 2015, doi: 10.1016/j.ress.2015.02.007.

[47] M. El-Gheriani, F. Khan, D. Chen, and R. Abbassi, "Major accident modelling using spare data," Process Saf. Environ. Prot., vol. 106, pp. 52-59, 2017, doi: 10.1016/j.psep.2016.12.004.

[48] M. Arif, F. Khan, S. Ahmed, and S. Imtiaz, "Rare event risk analysis - application to iceberg collision," J. Loss Prev. Process Ind., vol. 66, no. May, p. 104199, 2020, doi: 10.1016/j.jlp.2020.104199.

[49] M. Galagedarage Don and F. Khan, "Dynamic process fault detection and diagnosis based on a combined approach of hidden Markov and Bayesian network model," Chem. Eng. Sci., vol. 201, pp. 82-96, 2019, doi: 10.1016/j.ces.2019.01.060.

[50] C. Guo, F. Khan, and S. Imtiaz, "Copula-based Bayesian network model for process system risk assessment,” Process Saf. Environ. Prot., vol. 123, pp. 317-326, 2019, doi: 10.1016/j.psep.2019.01.022.

[51] B. Khan, F. Khan, and B. Veitch, "A Dynamic Bayesian Network model for ship-ice collision risk in the Arctic waters," Saf. Sci., vol. 130, no. May, p. 104858, 2020, doi: 10.1016/j.ssci.2020.104858.

[52] P. Yontay and R. Pan, "A computational Bayesian approach to dependency assessment in system reliability," Reliab. Eng. Syst. Saf., vol. 152, pp. 104-114, 2016, doi: 10.1016/j.ress.2016.03.005.

[53] R. Pan and P. Yontay, "Reliability assessment of hierarchical systems with incomplete mixed data," IEEE Trans. Reliab., vol. 66, no. 4, pp. 1036-1047, 2017, doi: 10.1109/TR.2017.2760802.

[54] L. Yang, K. He, Y. Du, Y. Guo, and P. Wang, "Reliability analysis to a satellite-equipped harmonic gear drive subjected to multisource data and imbalanced information," Int. J. Aerosp. Eng., vol. 2018, 2018, doi: $10.1155 / 2018 / 4256302$.

[55] L. Yang, Y. Guo, Q. Wang, and Z. Kong, "Reliability assessment of a complex system with unspecified structure and overlapping test data,” 2020, doi: 10.3850/978-981-11-2724-30889-cd. 